\title{
Outer radiation belt and inner magnetospheric response to sheath regions of coronal mass ejections: a statistical analysis
}

\author{
Milla M. H. Kalliokoski ${ }^{1}$, Emilia K. J. Kilpua ${ }^{1}$, Adnane Osmane ${ }^{1}$, Drew L. Turner ${ }^{2}$, Allison N. Jaynes ${ }^{3}$, Lucile Turc ${ }^{1}$, \\ Harriet George ${ }^{1}$, and Minna Palmroth ${ }^{1,4}$ \\ ${ }^{1}$ Department of Physics, Faculty of Science, University of Helsinki, Helsinki, Finland \\ ${ }^{2}$ Space Sciences Department, The Aerospace Corporation, El Segundo, California, USA \\ ${ }^{3}$ Department of Physics and Astronomy, University of Iowa, Iowa City, Iowa, USA \\ ${ }^{4}$ The Finnish Meteorological Institute, Helsinki, Finland
}

Correspondence: Milla M. H. Kalliokoski (milla.kalliokoski@ helsinki.fi)

Received: 25 October 2019 - Discussion started: 6 November 2019

Revised: 21 April 2020 - Accepted: 27 April 2020 - Published: 9 June 2020

\begin{abstract}
The energetic electron content in the Van Allen radiation belts surrounding the Earth can vary dramatically at several timescales, and these strong electron fluxes present a hazard for spacecraft traversing the belts. The belt response to solar wind driving is, however, largely unpredictable, and the direct response to specific large-scale heliospheric structures has not been considered previously. We investigate the immediate response of electron fluxes in the outer belt that are driven by sheath regions preceding interplanetary coronal mass ejections and the associated wave activity in the inner magnetosphere. We consider the events recorded from 2012 to 2018 in the Van Allen Probes era to utilise the energy- and radial-distance-resolved electron flux observations of the twin spacecraft mission. We perform a statistical study of the events by using the superposed epoch analysis in which the sheaths are superposed separately from the ejecta and resampled to the same average duration. Our results show that the wave power of ultra-low frequency Pc5 and electromagnetic ion cyclotron waves, as measured by a Geostationary Operational Environmental Satellite (GOES), is higher during the sheath than during the ejecta. However, the level of chorus wave power, as measured by the Van Allen Probes, remains approximately the same due to similar substorm activity during the sheath and ejecta. Electron flux enhancements are common at low energies $(<1 \mathrm{MeV})$ throughout the outer belt $(L=3-6)$, whereas depletion predominantly occurs at high energies for high radial distances $(L>4)$. It is distinctive that the depletion extends to lower energies at larger distances. We suggest that this $L$-shell and
\end{abstract}

energy-dependent depletion results from the magnetopause shadowing that dominates the losses at large distances, while the wave-particle interactions dominate closer to the Earth. We also show that non-geoeffective sheaths cause significant changes in the outer belt electron fluxes.

\section{Introduction}

The Van Allen radiation belts are toroidal regions surrounding the Earth that trap charged particles in the geomagnetic field (e.g. Van Allen, 1959). Traditionally the belts are divided into two zones of energetic populations, namely the relatively stable inner belt that is dominated by high-energy protons (e.g. Albert et al., 1998; Selesnick et al., 2016) and the electron-dominated outer belt in which electron fluxes vary widely - both temporally and spatially (e.g. Reeves et al., 2003; Baker et al., 2014b; Turner et al., 2014). This variability is driven by geomagnetic storm and substorm processes, by changes in the inner magnetospheric conditions, and by wave activity. These processes are initiated by solar wind energy input and disturbances in the solar wind that impact the Earth's magnetosphere (e.g. Daglis et al., 2019). The inner and outer belts are separated by a slot region at about 2 to 3 Earth radii, which is characterised by its low flux levels, though it can be flooded by electrons during storms (e.g. Baker et al., 2004).

The Van Allen belts expose spacecraft travelling beyond the low Earth orbit to hazardous radiation (e.g. Feynman and 
Gabriel, 2000; Horne and Pitchford, 2015; Green et al., 2017; Hands et al., 2018), and the geostationary orbit favoured by telecommunication and navigation satellites resides at the outer edge of the outer belt. While inner zone protons impose the most dangerous single effects, prolonged exposure to high-energy electrons due to possible sudden enhancements in the highly time-varying outer radiation belt is a significant space-weather-related threat for satellite operation. The increasingly popular nanosatellites, which often have less shielding available than larger spacecraft, are especially vulnerable to the bombardment of energetic particles in the radiation belts.

The electron fluxes in the outer radiation belt vary on timescales from minutes to days as a result of the different acceleration, transport, and loss processes. Wave-particle interactions play a key role in the electron dynamics and outer belt response to geomagnetic disturbances (e.g. Thorne, 2010). Both our capability to forecast the outer radiation belt dynamics and our understanding of the competing and coupled belt processes are still incomplete. Detailed studies focusing on how radiation belts respond to different solar wind drivers can shed light on the prompt evolution of the outer belt and improve the forecasting models used by the satellite industry.

Outer belt electrons are usually divided into the following different populations based on their energy: source (tens of kiloelectron volts), seed (hundreds of kiloelectron volts), and core (mega-electron volts). The highest energy population $(>\sim 3 \mathrm{MeV})$ is referred to as ultrarelativistic electrons. Source and seed electrons can originate from substorm injections, and the source population excites very low frequency (VLF) chorus waves that can, in turn, progressively accelerate seed electrons to relativistic energies (Jaynes et al., 2015). Chorus waves may also scatter the outer belt electrons into the loss cone (e.g. Bortnik and Thorne, 2007). Another important wave mode that changes the outer belt electron fluxes is ultra-low frequency (ULF) waves. The ULF-Pc5 pulsations (frequency range $2-7 \mathrm{mHz}$ ) are generated, for example, by Kelvin-Helmholtz instability at the magnetopause flanks (Claudepierre et al., 2008; Wang et al., 2017); by shocks and pressure pulses in the solar wind (Kepko and Spence, 2003; Claudepierre et al., 2010); and by perturbations in the ion foreshock (Hartinger et al., 2013; Wang et al., 2017). The ULF-Pc5 waves can lead to inward or outward radial diffusion of electrons, which results in acceleration or losses, respectively (Su et al., 2015; Shprits et al., 2006). On the other hand, electromagnetic ion cyclotron (EMIC) waves, which are generated by the temperature anisotropy of ring current protons, contribute to outer belt losses via resonant pitchangle scattering that lead to electron precipitation into the atmosphere (Usanova et al., 2014; Blum et al., 2019). The ULF-Pc5 waves can modulate the precipitation, for example, by lowering the mirror point of electrons (Brito et al., 2012) or by decreasing the minimum energy for resonance with EMIC waves (Zhang et al., 2019). Incoherent plasmaspheric hiss also scatters electrons and is thus important for the formation of the quiet-time slot region (e.g. Abel and Thorne, 1998; Jaynes et al., 2014).

The response of the outer radiation belt to geomagnetic storms has been studied extensively. These studies considered the response that is generally due to storm events (e.g. O'Brien et al., 2001; Reeves et al., 2003; Anderson et al., 2015; Turner et al., 2015; Moya et al., 2017; Murphy et al., 2018) and investigated the significance of the different storm drivers (e.g. Kataoka and Miyoshi, 2006; Hietala et al., 2014; Kilpua et al., 2015, 2019b; Turner et al., 2019). The studies found that the response depends on both the electron energy and the radial distance from the Earth (i.e. $L$ shell, see Mcllwain, 1961) and that different storm drivers cause distinct responses. The source and seed populations are dominated by enhancement, which tends to occur throughout the outer belt for source electrons and usually at lower $L$ shells for seed electrons, whereas the response of relativistic electrons is more evenly divided between enhancement, depletion, and no-change events (Turner et al., 2015, 2019). Source and seed populations that have been enhanced due to substorm activity, along with the interplay of chorus waves and electrons at these energies, play a large role in the radiation belt dynamics (Bingham et al., 2018, 2019; Katsavrias et al., 2019a).

One of the most important drivers of geomagnetic activity is the interplanetary coronal mass ejections (ICMEs; e.g. Kilpua et al., 2017) that enable effective magnetic reconnection at the magnetopause when their magnetic field has a strong southward component. An ICME that is sufficiently faster than the preceding solar wind will create a shock in front of it, and the turbulent region between the shock front and ICME ejecta is called the sheath region. The shock, sheath, and ejecta of an ICME have distinct solar wind properties and magnetospheric impact (see the review by Kilpua et al., 2017). Sheaths are turbulent and compressed structures with large amplitude magnetic field variations and high dynamic pressure, while ejecta are typically characterised by a smoothly changing magnetic field direction and low dynamic pressure. The outer belt response to sheaths and ejecta has been studied separately and combined ("full ICME") by, for example, Kilpua et al. (2015) and Turner et al. (2019). They found that energetic electrons $(>1 \mathrm{MeV})$ are more likely to be depleted during geomagnetic storms driven only by sheaths or ejecta, while full-ICME events are more likely to result in enhancement at this energy level. Kilpua et al. (2019b) performed a case study of a complex driver that consists of multiple sheaths and ejecta. They found that sheaths, more than the ejecta, were associated with stronger wave activity in the inner magnetosphere.

However, in most previous studies only the moderate or stronger geomagnetic storms (disturbance storm time (Dst) or symmetric disturbance (SYM- $H$ ) minimum of $-50 \mathrm{nT}$ or less) were considered, and the belt response was computed over long time intervals, usually by excluding fluxes in a $24 \mathrm{~h}$ period centred around the Dst or SYM- $H$ minimum. Our study has focused on the immediate outer belt response to 
sheath regions and has also considered the weak and nonstorm events. Previous studies showed that large geomagnetic activity is not required for significant changes in the outer radiation belt electron fluxes (Schiller et al., 2014; Anderson et al., 2015; Katsavrias et al., 2015). Furthermore, the radiation belts are an open system that particles enter via injections to and exit through losses to the magnetopause and atmosphere. Thus, to account for the total energy budget in the inner magnetosphere, we need to quantify enhancement and losses in timescales shorter than $24 \mathrm{~h}$. This immediate response is fundamental for distinguishing the effects of ICME sheaths and ejecta and is critical for enhancing our understanding of the Earth's radiation belt environment.

In this study, we consider the changes in the outer radiation belt electron fluxes by only comparing the fluxes from a few hours before and after the sheath region. This is done in order to contrast our study with the previous outer belt response studies that investigated the changes using intervals up to a few days. We also comprehensively analyse, for the first time, the geospace response during sheath regions and compare it to the responses during the preceding solar wind and the trailing ejecta. This analysis includes geomagnetic activity indices, subsolar magnetopause and plasmapause locations, energy and $L$-shell-dependent outer belt electron fluxes, and inner magnetospheric wave activity (chorus, Pc5, EMIC, and hiss). In addition to stronger geomagnetic activity (SYM- $H_{\min }<-50 \mathrm{nT}$ ), our study includes sheaths that caused only a weak geomagnetic storm $\left(-30 \mathrm{nT}>\right.$ SYM- $\left.H_{\min }>-50 \mathrm{nT}\right)$ or no geomagnetic storm at all (SYM- $H>-30 \mathrm{nT})$.

The paper is organised as follows. Section 2 presents the in situ data sets and the methods employed in our statistical study. We describe an example event and detail our statistical results in Sect. 3. In Sect. 4 we conclude our study and discuss future possibilities.

\section{Data and methods}

\subsection{Data}

We considered 37 ICME-driven sheath regions in the Van Allen Probes era since the launch of the spacecraft in August 2012. The events were selected based on the sheath region list compiled by Palmerio et al. (2016) for the period 2012-2015, and we used the sheath list in Kilpua et al. (2019a) for the period 2016-2018. We also added three events in 2016 that were identified by a visual inspection of the solar wind data. The timing of the shocks (i.e. sheath region front boundary) has been taken from the University of Helsinki Heliospheric Shock Database (http://www. ipshocks.fi, last access: 1 June 2020), and the ejecta leading edge (i.e. sheath region end boundary) were adjusted to match the boundary between the turbulent and compressed sheath plasma and the ejecta. The typical properties of sheath regions and ejecta, and the challenges in determining the boundary timings, are discussed, for example, in Richardson and Cane (2010) and Kilpua et al. (2017) and the references therein. We only included the cases with well-defined sheath and ejecta boundaries in this study.

The solar wind data were obtained from the Wind spacecraft that monitors solar wind at Lagrangian point L1. We used measurements from the Magnetic Field Investigation (MFI; Lepping et al., 1995) and Solar Wind Experiment (SWE; Ogilvie et al., 1995) on Wind. The Wind data were time shifted to the bow shock nose. The geomagnetic activity indices were taken from the OMNI database. The Wind and OMNI data were obtained through the NASA Goddard Space Flight Center (NASA-GSFC) Coordinated Data Analysis Web (CDAWeb, https://cdaweb.gsfc.nasa.gov/ index.html/, last access: 1 June 2020).

The twin Van Allen Probes (formerly known as the Radiation Belt Storm Probes (RBSPs) and identified as RBSPA and RBSP-B) are on highly elliptical orbits traversing through the inner and outer radiation belts (Mauk et al., 2013). The outer belt electron flux is measured as a function of the radial distance and electron energy by the Magnetic Electron Ion Spectrometer (MagEIS; Blake et al., 2013) and Relativistic Electron Proton Telescope (REPT; Baker et al., 2013) in the Energetic Particle, Composition, and Thermal Plasma (ECT; Spence et al., 2013) instrument suite on board the RBSP spacecraft. The Magnetic Electron Ion Spectrometer (MagEIS) covers electron energies from $30 \mathrm{keV}$ to $1.5 \mathrm{MeV}$ (source, seed, and core populations), while the core and ultrarelativistic electron populations are monitored by the REPT, which covers energies from 1.8 to $6.3 \mathrm{MeV}$. In this study, we employed level-2 spin-averaged differential electron flux data. For MagEIS electron fluxes, we only used the background-corrected data (Claudepierre et al., 2015). The temporal resolution of these data is $11 \mathrm{~s}$. We note that there is considerable variability in the energy scale of the MagEIS energy channels from early in the mission up to September 2013 (Boyd et al., 2019). Our study includes 13 events during this period, and our results could be slightly affected by these changes. We focused our study on the outer radiation belt between $L=2.5$ and $L=6$. The lower bound was chosen to avoid the proton contamination of the REPT in the inner belt, and the upper bound was constrained by the Van Allen Probes apogee. The $L$ parameter (Mcllwain, 1961), which was computed by using the TS04D magnetic field model (Tsyganenko and Sitnov, 2005), was extracted from the magnetic ephemeris data available on the ECT website (https://rbsp-ect.lanl.gov/, last access: 1 June 2020).

The very low frequency (VLF) wave activity in the inner magnetosphere, including chorus waves and plasmaspheric hiss, was obtained from the Electric and Magnetic Field Instrument Suite and Integrated Science (EMFISIS; Kletzing et al., 2013) on the Van Allen Probes. The utilised data product was the level-2 waveform receiver (WFR) diagonal spectral matrix, which contains the autocorrelations of electric 
and magnetic field components in 65 frequency bins. The frequency range spans from $2 \mathrm{~Hz}$ to $12 \mathrm{kHz}$, and the spectra are available with a $6 \mathrm{~s}$ time cadence. The EMFISIS team also provide electron densities estimated from the upper hybrid resonance frequency as level-4 data products (Kurth et al., 2015).

Additionally, observations of wave activity in the ultra-low frequency (ULF) range were taken from the GOES-15 spacecraft at geostationary orbit $(L \sim 6.6)$. The magnetic field data are sampled at $0.512 \mathrm{~s}$ by the GOES fluxgate magnetometers (Singer et al., 1996).

\subsection{Superposed epoch analysis}

In the superposed epoch analysis, the median of a given parameter is calculated from the data of all the events aligned with respect to some reference time (i.e. the zero epoch time). This technique has been used in various studies to investigate solar wind properties, wave activity, and electron fluxes statistically, for example (e.g. O'Brien et al., 2001; Kataoka and Miyoshi, 2006; Kilpua et al., 2013, 2015; Hietala et al., 2014; Murphy et al., 2018; Turner et al., 2019). We chose the zero epoch time to be at the shock and set an additional reference time at the ICME ejecta leading edge (i.e. at the end of the sheath region). We resampled the data during sheath regions to unify their durations. This double-epoch analysis allows us to study the general trends in the solar wind parameters and inner magnetospheric activity during driver structures that cover a large range of durations (for similar methods see, e.g., Kilpua et al., 2015; Masías-Meza et al., 2016; Yermolaev et al., 2018). The duration of the studied sheath regions varied widely, from 3.0 to $22.7 \mathrm{~h}$, with a standard deviation of $5.3 \mathrm{~h}$. The mean sheath duration was $12.0 \mathrm{~h}$. We resampled the sheath regions to match this mean sheath duration (Kilpua et al., 2013; Hietala et al., 2014). First, the data during the sheath were rescaled to start at 0 and end at $12.0 \mathrm{~h}$, and then the data were linearly interpolated to share the same time step in each event. For data that can vary over orders of magnitude (i.e. electron fluxes and wave power), we linearly interpolated the logarithm of these data. We note that some fluctuations can be averaged out when stretching or compressing the sheaths with linear interpolation. However, this should not significantly affect the results; the superposed epoch analysis of sheaths, with durations close to the mean duration of $12.0 \mathrm{~h}$ and ranging from 10 to $14 \mathrm{~h}$ (not shown), presented trends that are similar to the full set of events. The superposed epoch analysis was performed for geomagnetic indices, solar wind parameters, inner magnetospheric wave activity, and electron flux in the heart of the outer radiation belt $(L=3.5-5)$.

We considered wave activity in the very low and ultra-low frequency ranges in the superposed epoch analysis. Chorus waves appear outside the plasmasphere (where plasma density is approximately $<50-100 \mathrm{~cm}^{-3}$ ) in the following two distinct frequency bands (Burtis and Helliwell, 1969; Koons and Roeder, 1990): the lower band (0.1-0.5 $\left.f_{\mathrm{ce}}\right)$ and the upper band $\left(0.5-0.8 f_{\mathrm{ce}}\right)$, where $f_{\mathrm{ce}}$ is the electron cyclotron frequency. Plasmaspheric hiss waves occur inside the plasmasphere in a frequency range from about $100 \mathrm{~Hz}$ to $0.1 f_{\text {ce }}$. We included a study of hiss waves for completeness but we note that the timescales they operate on in outer belt electrons (> 2d; Jaynes et al., 2014) are longer than the sheath durations. We calculated the electron cyclotron frequency $f_{\mathrm{ce}}$ based on the TS04D geomagnetic field model (Tsyganenko and Sitnov, 2005). To determine whether the spacecraft was located inside or outside the plasmasphere at the time of the wave measurement, we estimated the plasmapause location with the auroral electrojet (AE)-index-based and magnetic local time (MLT)-dependent model by O'Brien and Moldwin (2003). A plasmapause model was used because the density estimate data are sporadic.

For ultra-low frequency waves, we calculated the wave power spectral density with a wavelet analysis from the magnetic field magnitude measured by GOES-15 at geostationary orbit. We calculated the Pc5 wave power in the range from 2.5 to $10 \mathrm{~min}(2-7 \mathrm{mHz})$ and the EMIC wave power in the range from 0.2 to $10 \mathrm{~s}(0.1-5 \mathrm{~Hz})$, which corresponds to the range of Pc1 and Pc2 pulsations given by Jacobs et al. (1964). The power spectral densities were averaged in the given frequency ranges of the wave modes to obtain the wave power data for the superposed epoch analysis, and resampling was performed after this averaging. We note that the GOES measurements taken at geostationary orbit might not always reflect the ULF-Pc5 and EMIC wave activity at the position of the Van Allen Probes (Engebretson et al., 2018; Georgiou et al., 2018).

For the electron flux in the superposed epoch analysis, we considered the median flux in the heart of the outer belt at $L=3.5-5$. The MagEIS and REPT electron flux measurements were binned in time $(\Delta t=4 \mathrm{~h})$ and $L$ shell $(\Delta L=$ $0.1)$ to combine the data from the two spacecraft. The $4 \mathrm{~h}$ cadence was chosen to reduce the effect of the Van Allen Probes orbits and in order to minimise the data gaps in the binned flux data during all events. We note that a $4 \mathrm{~h}$ cadence leaves us with only 4 data points during the sheath, but here we are mostly interested in the overall trend during the events, which is similar at higher time resolutions. We selected four energy channels to represent the source $(54 \mathrm{keV})$, seed $(346 \mathrm{keV})$, core $(1064 \mathrm{keV})$, and ultrarelativistic $(4.2 \mathrm{MeV})$ populations. We also calculated the mean electron flux at $L=3.5-5$ with the same time and $L$-shell bins (not shown), and we noted that the trends are very similar to the median values.

In addition to investigating the median sheath properties of all 37 events, we divided the events based on the level of associated geomagnetic activity inferred from the SYM$H$ index. The SYM- $H$ index (Iyemori, 1990; Iyemori and Rao, 1996) is derived from perturbations in the horizontal component $(H)$ of the geomagnetic field that is affected by changes in the ring current. The SYM- $H$ index is essentially 
the same as the hourly Dst index but with a higher time resolution of $1 \mathrm{~min}$, and it is also more sensitive to substorm activity. The strength of a geomagnetic storm is usually characterised by the minimum Dst value, where the thresholds for small, moderate, and intense storms are $-30,-50$, and $-100 \mathrm{nT}$, respectively (Gonzalez et al., 1994). In previous studies, typically only moderate or larger storms with Dst or SYM- $H$ less than $-50 \mathrm{nT}$ were considered (e.g. O'Brien et al., 2001; Reeves et al., 2003; Kilpua et al., 2015; Lugaz et al., 2016; Turner et al., 2015, 2019). However, due to the relatively low number of well-defined sheath events during Van Allen Probes measurements, only 9 events out of our total of 37 events have a SYM- $H$ minimum below $-50 \mathrm{nT}$ during the sheath region or $2 \mathrm{~h}$ afterwards. Therefore, we set the threshold to $-30 \mathrm{nT}$ to obtain a statistically adequate subset of 17 geoeffective events. The interval from which we took the minimum was extended to $2 \mathrm{~h}$ after the sheath to accommodate for lag in the ring current response. Note that the geomagnetic disturbance of the ICME ejecta was not considered.

\subsection{Electron flux response}

We binned the MagEIS and REPT spin-averaged electron flux data from both spacecraft in $0.1 \mathrm{~L}$-shell bins and $1 \mathrm{~h}$ time bins, which differed from the superposed epoch analysis, in order to have a higher time resolution. Based on the methodology of Reeves et al. (2003) and Turner et al. (2015, 2019), we define the outer belt electron response $(R)$ as the ratio of post-event flux to pre-event flux. The pre-event flux was obtained by averaging the electron flux over a $6 \mathrm{~h}$ interval before the sheath region, and the post-event flux was obtained by averaging over $6 \mathrm{~h}$ after the sheath region. The response parameter $R$ was computed for each electron energy and $L$ shell bin considered. The response was categorised as enhancement when the post-event flux had increased by over a factor of 2 when compared to the pre-event flux $(R>2), d e$ pletion when it had decreased by over a factor of $2(R<0.5)$, and no change when the flux level had not changed significantly $(0.5 \leq R \leq 2)$.

In previous studies (Reeves et al., 2003; Turner et al., 2015, 2019), the pre- and post-event fluxes were defined as the maximum flux from $>12 \mathrm{~h}$ up to a few days before and after the event because the outer radiation belt response to entire geomagnetic storms was examined. These studies also excluded the $24 \mathrm{~h}$ period during the storm. In this study we use the mean flux values close to the sheath region because we only focus on the outer belt response to the sheath region, and all sheaths did not generate geomagnetic storms (in 20 out of 37 events the SYM- $H$ index does not drop below $-30 \mathrm{nT}$ ). A post-sheath maximum flux value is not meaningful because it would be embedded in the ICME ejecta and subject to possible fluctuations driven by the ejecta. The $6 \mathrm{~h}$ averaging period aims to capture the changes generated by the sheath while excluding the main response to the ejecta, which is expected to occur later (mean duration of the ejecta was $28.4 \mathrm{~h}$, with a standard deviation of $11.1 \mathrm{~h}$ ).

\section{Results}

\subsection{Example event on 7 February 2014}

Figure 1 shows the solar wind conditions and geomagnetic indices when an interplanetary coronal mass ejection (ICME) driving a sheath region impacted the Earth during 7-9 February 2014. The shock (first red vertical line in Fig. 1) was identified as an abrupt and simultaneous increase in the magnetic field and solar wind speed, as well as a small increase in dynamic pressure. Both the sheath and ICME were relatively slow $\left(\approx 400-450 \mathrm{~km} \mathrm{~s}^{-1}\right)$. The shock was also quite weak, as the speed jump across the shock was about $100 \mathrm{~km} \mathrm{~s}^{-1}$. The sheath was characterised by a fluctuating magnetic field and variable dynamic pressure that had high values $(\approx 20 \mathrm{nPa})$ in the trailing half of the sheath. The ejecta had a smoother field and a clearly lower dynamic pressure. This ICME is included in the Richardson and Cane ICME list (http://www. srl.caltech.edu/ACE/ASC/DATA/level3/icmetable2.htm, last access: 1 June 2020; Richardson and Cane, 2010) and is recorded as a "magnetic cloud" there (i.e. the event shows signatures of a magnetic flux rope). This is because the magnetic field components (Fig. 1b) show some organised rotation during the ICME, and the north-south magnetic field component $\left(B_{Z}\right)$ rotates from north to south. In the sheath, the field was predominantly northward.

The event was only mildly geoeffective (Fig. If and g). The SYM- $H$ index dropped to $-29 \mathrm{nT}$ in the middle of the sheath (and briefly below $-30 \mathrm{nT} 1 \mathrm{~h}$ after the sheath ended), and the ICME only caused a weak storm. The substorm activity was also weak (but quite continuous) as shown in the substorm activity (AL) index.

Despite the low geoeffectiveness of both the sheath and ejecta, there were clear changes in the outer radiation belt electron fluxes at source, seed, core, and ultrarelativistic energies as shown in Fig. 2. Note that, for this particular event, background-corrected fluxes are not available for $L>3$ at source energies. Before the shock arrived, the outer belt showed typical undisturbed conditions (e.g. Reeves et al., 2016), and the seed and core electron fluxes were higher at the highest $L$ shells. The ultrarelativistic electrons, in turn, peaked at $L \sim 4$. After the shock arrival, the fluxes increased at seed and higher energies. The most distinct increase was detected in the ultrarelativistic $(4.2 \mathrm{MeV})$ energies. The fluxes at seed, core, and ultrarelativistic energies also widened towards lower $L$ shells during the sheath. The flux of the source population ( $54 \mathrm{keV}$ ) increased significantly at the end of the sheath. At higher energies, on the other hand, the flux was depleted near the sheath-ejecta boundary. Interestingly, the ultrarelativistic electron fluxes were already enhanced in the front part of the ICME ejecta after the sheath, 
while the seed and core electron fluxes clearly only increased near the middle of the ejecta.

The wave activity in the inner magnetosphere during the event is illustrated by Fig. 3, which shows the wave power spectral density of both the very low and ultra-low frequency waves as measured by RBSP-B and GOES-15, respectively. Some chorus activity (Fig. 3a) appeared immediately after the shock, and it was enhanced in the latter half of the sheath region. Chorus activity persisted during the ejecta. The chorus waves might have caused some acceleration - the enhancement of $1 \mathrm{MeV}$ electrons during the ejecta, for example - but the waves would not yet have acted long enough to cause the enhancement of ultrarelativistic electrons during the sheath (e.g. Jaynes et al., 2015).

The ULF wave power in the Pc5 and EMIC ranges was elevated during the sheath (Fig. 3f). The widening of the electron fluxes towards lower $L$ shells could thus be a result of inward radial transport by ULF waves (e.g. Turner et al., 2013; Jaynes et al., 2018). EMIC waves can also be responsible for the loss of relativistic electrons (Usanova et al., 2014). The subsolar magnetopause was located at about $12.7 R_{\mathrm{E}}$ before the shock arrival, according to the Shue et al. (1998) model (Fig. 1e). The shock pushed the magnetopause nose inward, and it was located closest to Earth $\left(\approx 7.5 R_{\mathrm{E}}\right)$ at the end of the sheath when the depletion occurred. At the ejecta leading edge, the magnetosphere started to recover and the subsolar magnetopause stayed at about $10 R_{\mathrm{E}}$ during the ejecta. Therefore, losses at the magnetopause (i.e. magnetopause shadowing) could be the main driver of the depletion, which was possibly coupled with outward transport by ULF waves (Turner et al., 2012). During the ejecta, the chorus waves continued but the ULF-Pc5 waves, and the EMIC wave activity in particular, weakened. The enhancement of seed and core fluxes in the ejecta was thus likely associated with continued chorus activity and possibly also with inward transport by ULF-Pc5 waves.

\subsection{Statistics of 37 sheath events}

Statistics from the superposed epoch analysis of 37 events with sheath regions are presented in Fig. 4. Results are also shown for $10 \mathrm{~h}$ of solar wind before the shock and for $1 \mathrm{~d}$ of the ICME ejecta after the sheath (note that, unlike the sheath regions, the ejecta were not resampled). The results in panels (a)-(d) show the general characteristics of sheath regions (e.g. Kilpua et al., 2017, 2019a) as follows: lower magnetic field magnitude than in the ejecta (but about twice as strong as in the quiet solar wind); elevated dynamic pressure (and solar wind density) compared to quiet solar wind conditions and the ejecta; and contracted magnetopause nose due to the high dynamic pressure sheath. The SYM- $H$ index usually has a positive peak at the shock, which corresponds to the storm's sudden commencement and initial phase or sudden impulse, and then it gradually decreases during the sheath (Fig. 4e). However, on average, the main geomagnetic storm

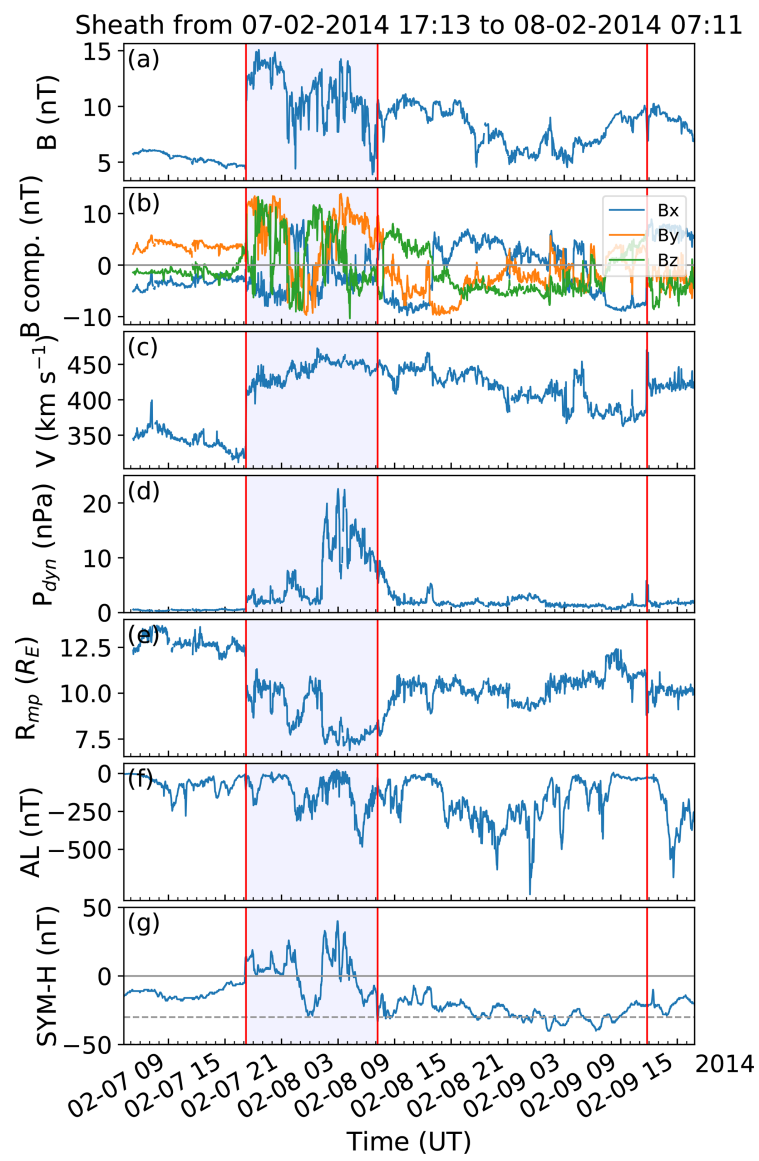

Figure 1. (a) Magnetic field magnitude, (b) magnetic field components in the geocentric solar magnetospheric coordinate system, (c) solar wind speed, (d) solar wind dynamic pressure, (e) subsolar magnetopause location from the Shue et al. (1998) model, (f) AL index, and (g) SYM- $H$ index. The red vertical lines indicate the shock, ICME ejecta leading edge, and ejecta trailing edge in UT (universal time). The shaded area marks the sheath interval. The dashed line in panel $(\mathbf{g})$ indicates SYM- $H=-30 \mathrm{nT}$. Note: times are shown in the format of $\mathrm{mm} / \mathrm{dd}$ hh.

impact is imposed by the ejecta. In 17 events, the SYM- $H$ index dropped below $-30 \mathrm{nT}$ (weak storm) during the sheath or $2 \mathrm{~h}$ afterwards, and it dropped below $-50 \mathrm{nT}$ (moderate storm) in only 9 events. On average, only weak substorm activity is evidenced by the AL index during the sheath region and ejecta (Fig. 4f).

Panels (g)-(l) of Fig. 4 show the statistics of different wave modes in the inner magnetosphere during the selected events. The ULF-Pc5 wave power peaks in the sheath, which shows a growing trend from the shock towards the end of the sheath region (Fig. 4g). The mean of the median Pc5 wave power in the sheath is about $10^{2} \mathrm{nT}^{2} \mathrm{~Hz}^{-1}$, as measured by GOES-15 at $L \sim 6.6$, which is 1 order of magnitude larger than during quiet solar wind. The wave power of Pc5 waves declines during the ejecta. The EMIC wave power is also larger in the sheath than during the ejecta and quiet solar wind, with a 

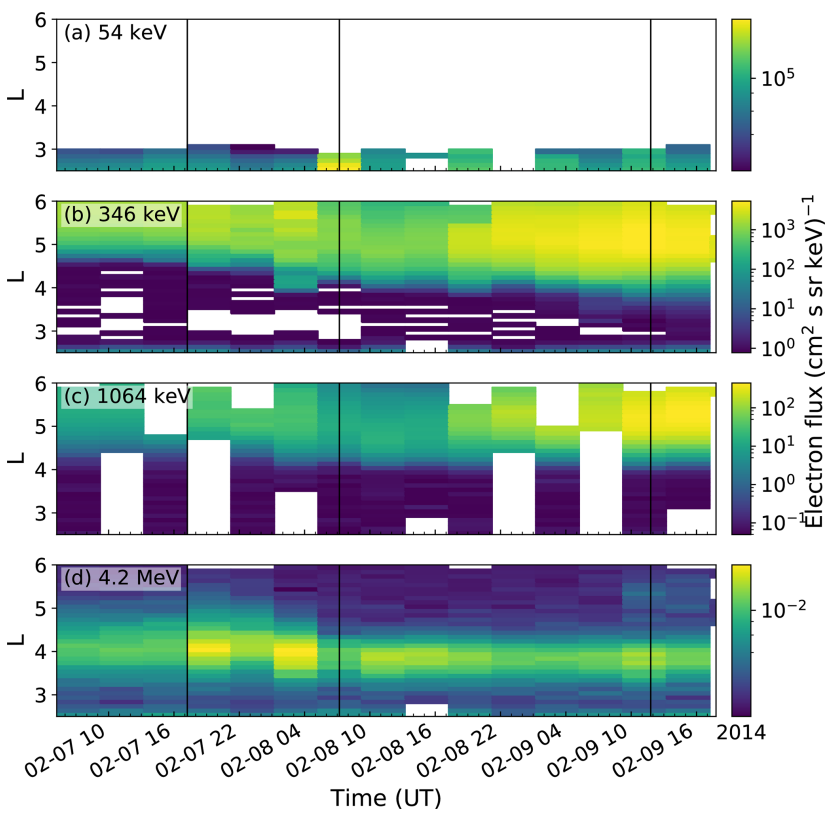

Figure 2. The spin-averaged electron fluxes measured by MagEIS at (a) $54 \mathrm{keV}$ (source), (b) $346 \mathrm{keV}$ (seed), and (c) $1064 \mathrm{keV}$ (core) and by REPT at (d) $4.2 \mathrm{MeV}$ (ultrarelativistic). The data are combined from both Van Allen Probes and are binned by $4 \mathrm{~h}$ in time and 0.1 in $L$ shell. The vertical lines mark the sheath region and ICME ejecta intervals. Note: times are shown in the format of $\mathrm{mm} / \mathrm{dd} \mathrm{hh}$.

median wave power of about $10^{-2.5} \mathrm{nT}^{2} \mathrm{~Hz}^{-1}$ (Fig. 4h). The median EMIC wave power quickly decreases to the pre-event level of about $10^{-3} \mathrm{nT}^{2} \mathrm{~Hz}^{-1}$ in the ejecta.

The main power of chorus waves is in the lower band, where the order of magnitude during the sheath is $10^{-9} \mathrm{nT}^{2} \mathrm{~Hz}^{-1}$ (Fig. 4j). The median wave power of the upper-band chorus is 1 order of magnitude lower, but in a quarter of the cases the power can reach values comparable to lower-band waves as shown in the upper-quartile curve (Fig. 4k). The chorus wave power is very similar in the sheath and the ejecta, and it is also only about 4 times higher on average during the sheath than during the pre-event conditions. The chorus wave power increases gradually for a few hours before the shock arrival. This could be driven by the very weak substorm activity in front of the ICME event. Plasmaspheric hiss is not affected by the sheath or ejecta, and its median wave power remains at about $10^{-8} \mathrm{nT}^{2} \mathrm{~Hz}^{-1}$ throughout the event (Fig. 41).

The behaviour of the median electron fluxes in the heart of the outer belt $(L=3.5-5)$ is shown in panels $(\mathrm{m})-(\mathrm{p})$ of Fig. 4. The flux of the source population increases during the sheath region and it mostly stays at a constant level during the ejecta. A comparison of the pre-sheath to post-sheath fluxes shows that the median response of $54 \mathrm{keV}$ electrons is an enhancement by a factor of 5 . For the seed population, the flux is slightly enhanced at the shock but, on average, the flux remains unaffected in the sheath. However, the flux suddenly
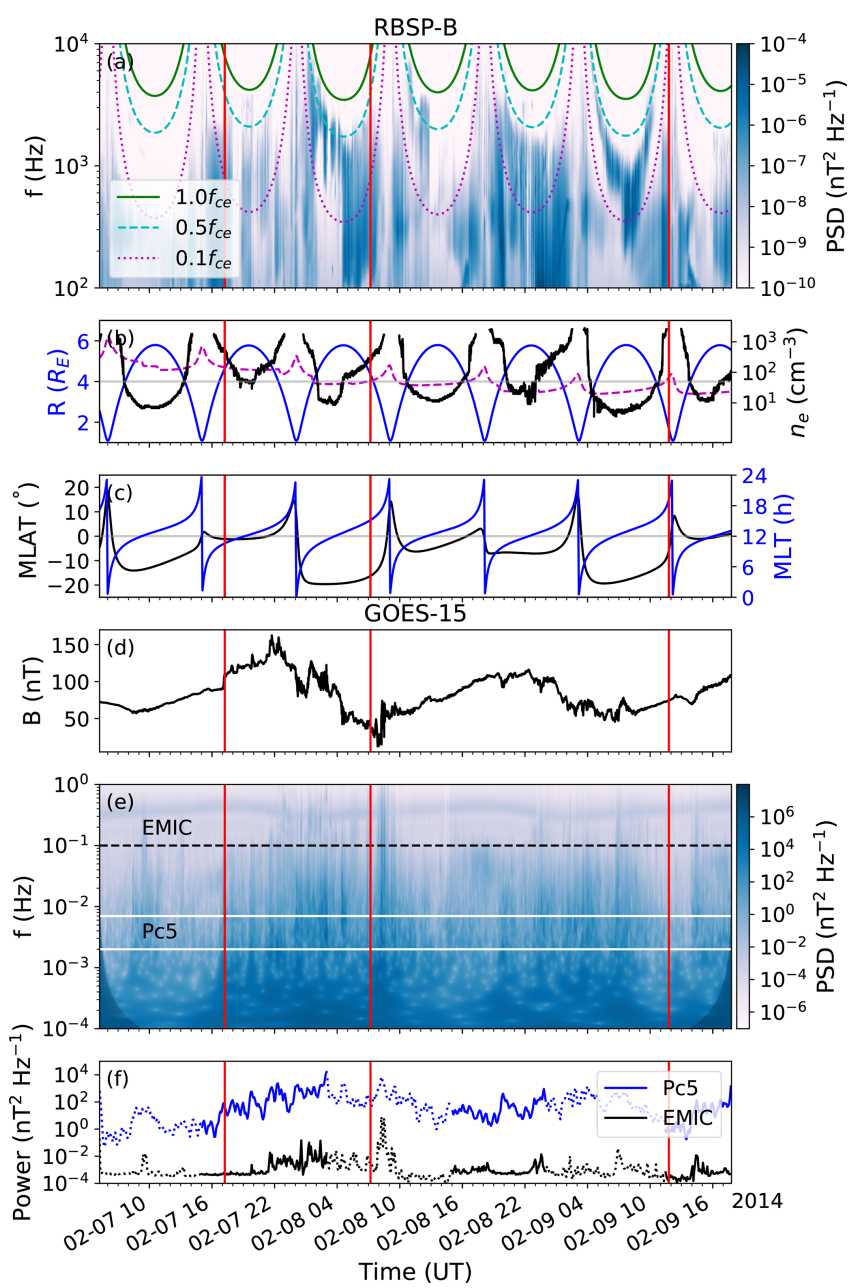

Figure 3. Very low frequency (VLF) and ultra-low frequency (ULF) wave activity. (a) Power spectral density of VLF waves from RBSP$\mathrm{B} / \mathrm{EMFISIS}$. The curves indicate different values of the equatorial gyrofrequency $f_{\text {ce }}$ calculated from the TS04D geomagnetic field model. Chorus waves have frequencies $>0.1 f_{\text {ce }}$ outside the plasmasphere, and the plasmaspheric hiss is present at lower frequencies. (b) TS04D model spacecraft radial location in blue, with the model plasmapause location (O'Brien and Moldwin, 2003) shown as a dashed magenta line (left axis) and estimated electron density (right axis). The horizontal line at $50 \mathrm{~cm}^{-3}$ illustrates another estimate of the plasmapause location. (c) TS04D model magnetic latitude (left axis) and magnetic local time (right axis). (d) Magnitude of the magnetic field as measured by GOES-15. (e) Power spectral density of ULF waves from wavelet analysis of the GOES-15 magnetometer measurements. The shaded areas mark the cone of influence. The white horizontal lines indicate the range of the Pc5 pulsations, and the dashed horizontal line indicates the lower boundary of the EMIC range. (f) Wave power of ULF-Pc5 and EMIC waves. Solid and dotted lines indicate when GOES-15 was on the dayside and nightside, respectively. The red vertical lines indicate the sheath and ICME ejecta intervals. Note: times are shown in the format of $\mathrm{mm} / \mathrm{dd} \mathrm{hh}$ 
increases after the sheath ends and continues to be enhanced in the ejecta. The $346 \mathrm{keV}$ electron median flux increases by a factor of about 25 . At MeV energies, the flux in the heart of the outer belt remains mostly unchanged when considering the median response. The flux in the ejecta is less than a factor of 2 larger than before the sheath, based on the medians for both $1064 \mathrm{keV}$ and $4.2 \mathrm{MeV}$. However, the upper quartile of the core population at $1064 \mathrm{keV}$ shows a slight increase and the lower quartile a slight decrease, thereby evidencing that these opposite responses are averaged out in the median. Both the upper and lower quartiles for $4.2 \mathrm{MeV}$ electrons indicate a slight decrease in the flux.

The superposed epoch analysis of the plasmapause location from an AE-index-based model (O'Brien and Moldwin, 2003) is shown in Fig. 5 as both independent of magnetic local time (MLT) throughout the event and as MLTdependent for the pre-event time ( $-6 \mathrm{~h}$ from shock), sheath region $(+6 \mathrm{~h})$, and ejecta $(+18 \mathrm{~h})$. One event in 2018 was excluded from the analysis due to the $\mathrm{AE}$ index data not being available. The MLT dependence of the model shows that the plasmapause is closer to the Earth on the dayside and further away on the nightside during both quiet and disturbed times. In the preceding solar wind, the plasmapause is located at about $5 R_{\mathrm{E}}$. During the sheath, the plasmasphere moves earthward, and it moves even further earthward during the ejecta. The variation is consistent with the general $\mathrm{AL}$ levels in the preceding solar wind, sheath, and ejecta (the AE index should roughly follow AL behaviour). At noon MLT, the median plasmapause location moves from about $4.4 R_{\mathrm{E}}$ during the quiet solar wind conditions to $3.6 R_{\mathrm{E}}$ in the middle of the sheath. At $6 \mathrm{~h}$ after the sheath region, i.e. $18 \mathrm{~h}$ after the shock, the median distance has decreased to $3.3 R_{\mathrm{E}}$.

The electron flux response of the whole outer radiation belt for a wider selection of energies than in the superposed epoch analysis is shown in Fig. 6, where the response is divided to the three categories of enhancement, depletion, and no change. The source population at $L>3.5$ is enhanced in $80 \%$ of the cases and is practically never depleted. Moreover, closer to the inner boundary of the outer belt, the nochange events are very common at all energies. Electrons at seed energies are enhanced in about half of the cases throughout the belt, with a higher possibility for enhancement in the heart of the outer belt. In a small subset $(<15 \%)$ of the seed electrons, depletion occurs near $L \sim 3$. Depletion is most common in the $\sim 1-3 \mathrm{MeV}$ core population at high $L$ shells ( $L>4.5$ ). At lower $L$ shells, core electron flux is enhanced in $10 \%$ of the cases at most, and in a major fraction of the events $(>60 \%)$ the core electron fluxes do not change significantly below $L \sim 4.5$.

Interestingly, a feature of the outer belt response is that the depletion progresses to lower energies when $L$ increases. At $L \sim 4.5$ the depletion dominates only at $>2 \mathrm{MeV}$ energies, while at $L \sim 6$ it extends to seed energies at around $500 \mathrm{keV}$.

\subsection{Impact of geoeffectiveness}

After dividing the 37 studied sheath events based on the geomagnetic disturbance they cause, which was inferred from the SYM- $H$ index ( $\leq-30 \mathrm{nT}$ for geoeffective events) during the sheath region and $2 \mathrm{~h}$ after it, we found a different response in the outer radiation belt. The superposed epoch analysis results presented in Figs. 7 and 8 show that geoeffective events are associated with larger dynamic pressure and magnetic field magnitude in the sheath and tend to have higher speeds. Geoeffective sheaths are also more often accompanied by strongly geoeffective ejecta than sheaths where the SYM- $H$ index remains close to $0 \mathrm{nT}$. Geoeffective sheaths also have larger positive SYM- $H$ peaks at the shock, which is likely due to their tendency for high dynamic pressure, and, as expected, the substorm activity is greater during geoeffective events as evidenced by the AL index.

The wave power of Pc5, EMIC, and chorus waves is higher by a factor of about 6 in geoeffective sheaths when compared to non-geoeffective ones. In geoeffective events, the jump in wave power at the shock is larger in all considered wave modes. For example, the median Pc5 wave power is about 50 times higher during the sheath than before the shock arrival in geoeffective events, whereas in non-geoeffective cases it is only about 20 times higher. The Pc5 wave power also gradually decreases in geoeffective ejecta, but it remains at an approximately constant level that is lower than the median power in the sheath during less-effective ejecta; however, the wave power has a slightly increasing trend near the end of the considered period. The median EMIC wave power behaves similarly between the two groups of events.

While the median chorus wave power in geoeffective events increases, on average, by 1 order of magnitude from pre-event conditions to the sheath region, the chorus activity does not differ significantly between the sheath and ejecta, where it is about $10^{-8} \mathrm{nT}^{2} \mathrm{~Hz}^{-1}$ for lower band and $10^{-9} \mathrm{nT}^{2} \mathrm{~Hz}^{-1}$ for upper band chorus. We also note that in a quarter of the geoeffective cases the upper band chorus wave power is significantly enhanced at the shock (Fig. 7k). Nongeoeffective sheaths that are associated with modest substorm activity drive the chorus waves in only about a quarter of the events, and the median chorus wave power remains at roughly the pre-event level throughout the ICME $\left(10^{-9} \mathrm{nT}^{2} \mathrm{~Hz}^{-1}\right.$ for lower band and $10^{-10} \mathrm{nT}^{2} \mathrm{~Hz}^{-1}$ for upper band chorus), which is opposed to the geoeffective events where substorm injections excite stronger chorus activity during the ICME. The median wave power of plasmaspheric hiss is, on average, twice as high during geoeffective events than during non-geoeffective events.

The median fluxes in the heart of the outer belt experience enhancement at all the considered energies in the geoeffective events. The strongest increase occurs in the seed population, whose median flux increases by almost 2 orders of magnitude. During the sheath the flux gradually increases at 

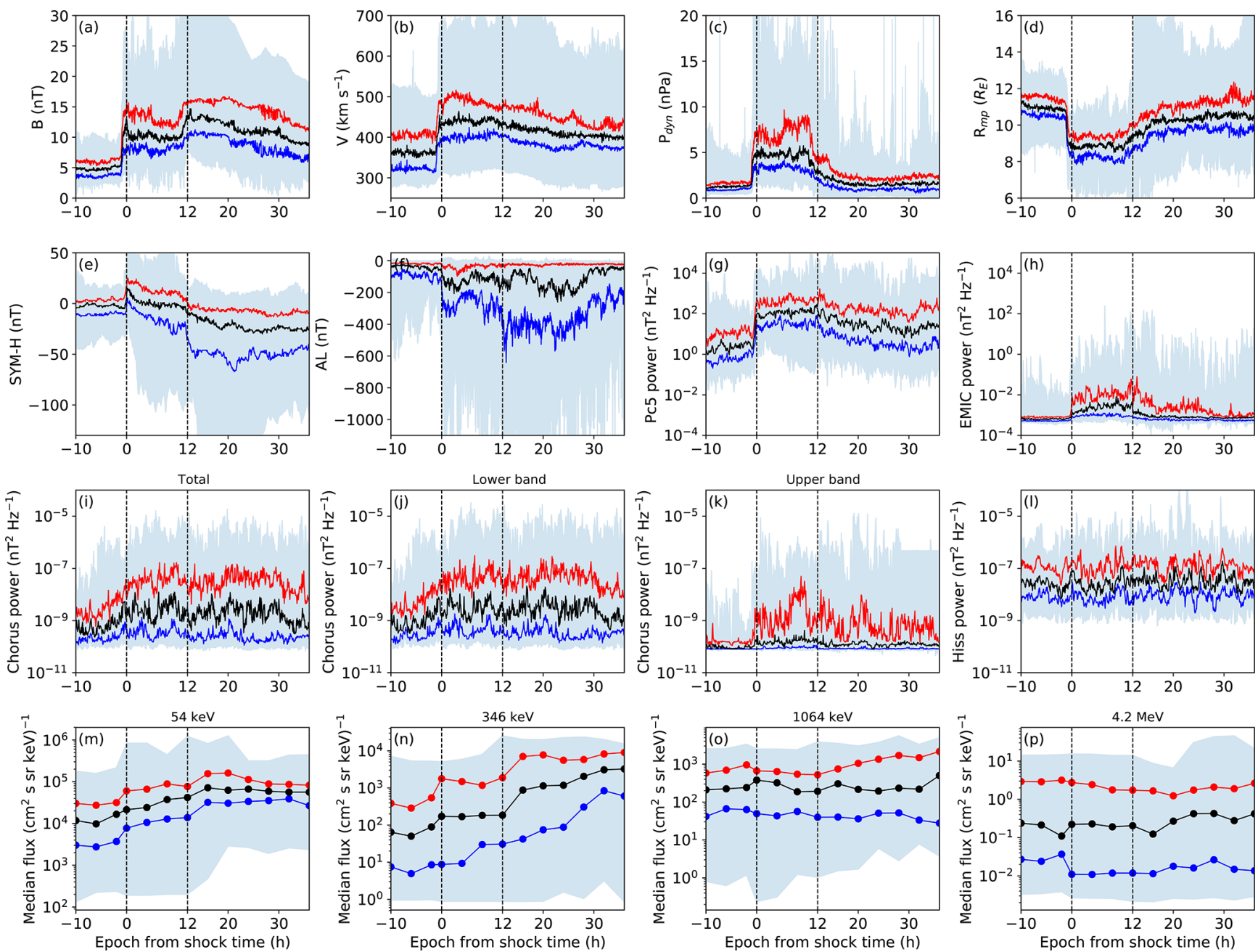

Figure 4. Results of the superposed epoch analysis for solar wind data, geomagnetic indices, VLF and ULF wave powers, and median electron fluxes in the heart of the outer radiation belt $(L=3.5-5)$. The black curves show the medians, and the red and blue curves show the upper and lower quartiles, respectively. The shaded area indicates the full range of data from all events. Panels show (a) interplanetary magnetic field magnitude, (b) solar wind speed, (c) solar wind dynamic pressure, (d) subsolar magnetopause location from the Shue et al. (1998) model, (e) SYM- $H$ index, and (f) AL index. Panels (g) and (h) show wave activity as ULF-Pc5 and EMIC wave power; (i)-(k) show total, lower band, and upper band chorus wave power; and (l) shows plasmaspheric hiss wave power. The median electron fluxes are shown in panels (m)-(p) at source $(54 \mathrm{keV})$, seed $(346 \mathrm{keV})$, core $(1064 \mathrm{keV})$, and ultrarelativistic $(4.2 \mathrm{MeV})$ energies, respectively. The wave power of ULF waves (Pc5 and EMIC) was computed from GOES-15 measurements, whereas VLF wave power (chorus and hiss) was obtained from RBSP-A and RBSP-B, with the plasmapause location taken into account (using the model by O'Brien and Moldwin, 2003).

the source and seed energies, while it decreases and is the lowest during the sheath at $\mathrm{MeV}$ energies.

For non-geoeffective events the source and seed populations are enhanced, but the flux at core energies does not change significantly and the ultrarelativistic population is depleted. This differs from the geoeffective case in which enhancement occurred at all four energies. The median electron flux at $54 \mathrm{keV}$ increases throughout the event, but the seed population at $346 \mathrm{keV}$ remains at the same level during the sheath after an initial large increase at the shock, and the flux begins to increase again after only a few hours in the ejecta. The $1064 \mathrm{keV}$ electron flux is slightly enhanced during the sheath before the depletion, while electron losses at $4.2 \mathrm{MeV}$ energies already take place at the shock. The change in median fluxes is also lower than in geoeffective events, with the largest change being an increase by a factor of 20 at seed energies in non-geoeffective events.

Again, the outer belt response as a function of $L$ shell and a wider range of electron energies is considered, and the results are shown separately for geoeffective and non-geoeffective events in Fig. 9. It is immediately evident that, for geoeffective sheaths, enhancement events are more common at all energies and $L$ shells, and the source and seed populations are practically always enhanced in the heart of the outer belt 
(a)

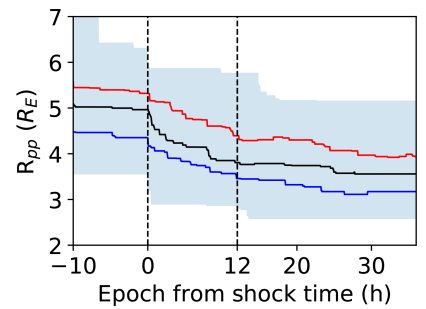

(b)

Time from shock

-6 hours (preceding solar wind)

6 hours (sheath)

18 hours (ejecta)

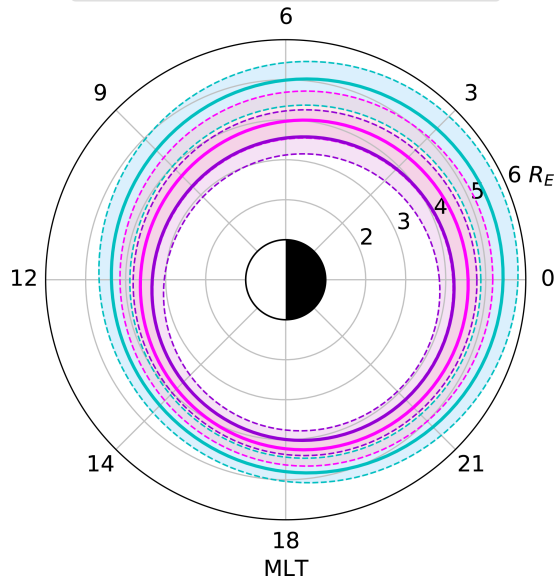

Figure 5. Results of the superposed epoch analysis for the model plasmapause location (O'Brien and Moldwin, 2003) for the nonMLT-dependent case and as a function of MLT. (a) The non-MLTdependent results are presented in the same format as the results in Fig. 4. (b) The MLT-dependent plasmapause is shown at $6 \mathrm{~h}$ before (cyan), $6 \mathrm{~h}$ after (magenta), and $18 \mathrm{~h}$ after (violet) the epoch time at the shock by sampling the pre-event, sheath, and ejecta regions, respectively. The medians are shown in solid lines, while the upper and lower quartiles are indicated with the dashed lines and shaded area.

( $L=3.5-5)$. However, $>\mathrm{MeV}$ electrons experience depletion more frequently in geoeffective events throughout the outer belt, which deviates from the superposed epoch analysis results. In non-geoeffective events depletion only begins to dominate the core population response at around $L>5$. Virtually all non-geoeffective events result in no significant change at low $L$ shells $(L<4.5)$ at almost all energies, while flux enhancements practically only take place at source energies and are limited to $L>4$.

\section{Discussion and conclusions}

In this paper we studied the inner magnetospheric wave activity statistically, in addition to the energy and $L$-dependent outer radiation belt electron flux response during ICMEdriven sheath regions. Our study included 37 sheaths during the Van Allen Probes era (2012-2018).

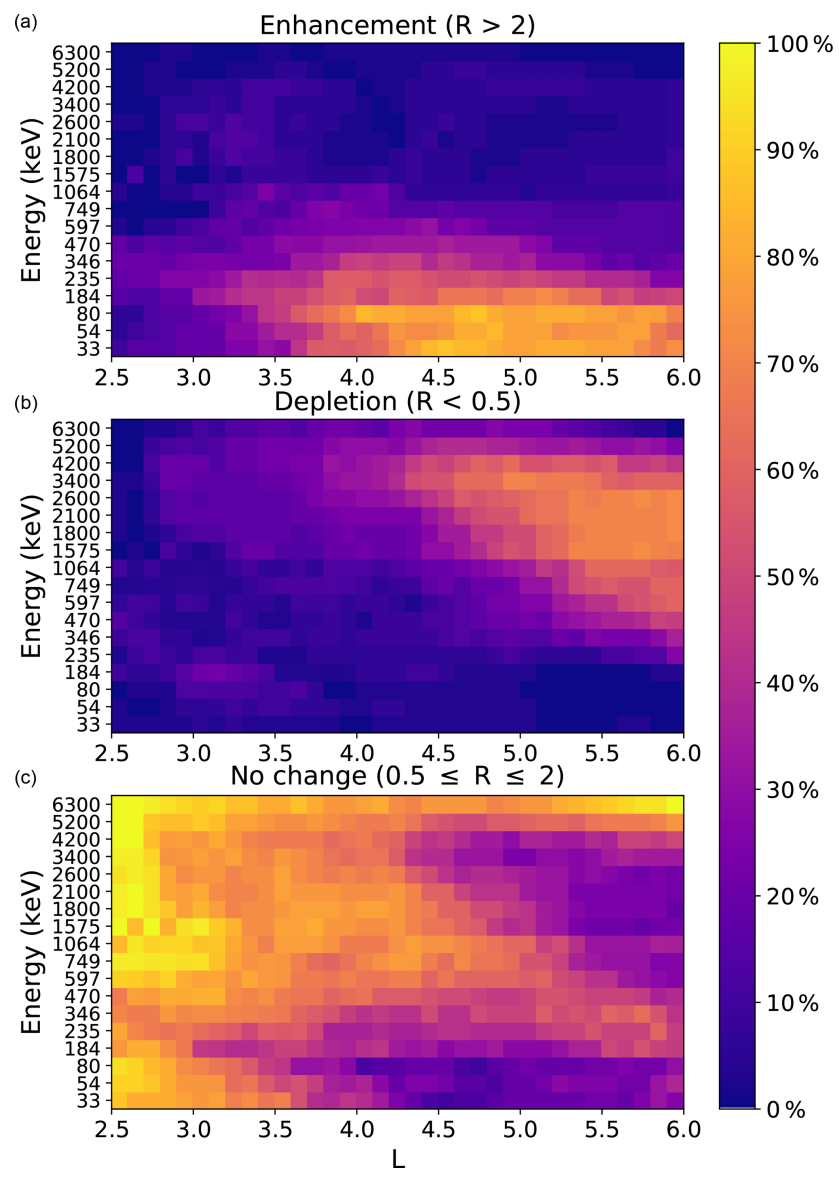

Figure 6. Percentage of the sheath events causing (a) enhancement, (b) depletion, or (c) no change in the outer radiation belt electron fluxes as a function of electron energy and $L$ shell ( 0.1 bins). The sum of percentages from all three panels for a given energy and $L$-shell bin is $100 \%$.

We found that turbulent sheath regions preceding ICMEs caused significant changes in the outer radiation belt electron fluxes. While the response was the most dramatic for geoeffective sheaths, we emphasise that these changes also occurred during the sheaths that only caused a weak geomagnetic storm or that were not geoeffective at all in terms of their SYM- $H$ response. These results are consistent with previous findings that have reported clear responses during small geomagnetic storms (Anderson et al., 2015) and also during non-geoeffective sheaths in case studies (e.g. Alves et al., 2016; Kilpua et al., 2019b). The ejecta in our data set had a larger SYM- $H$ response than the sheath regions.

Our analysis showed that the inner magnetospheric wave activity was clearly enhanced in the sheath when compared to the preceding solar wind, Pc5 wave power was enhanced by 1 order of magnitude, and EMIC and chorus wave power were 4 times higher than in the preceding solar wind. We also found that ULF-Pc5 and EMIC wave power were larger in the sheath than in the following ejecta. This is in agree- 

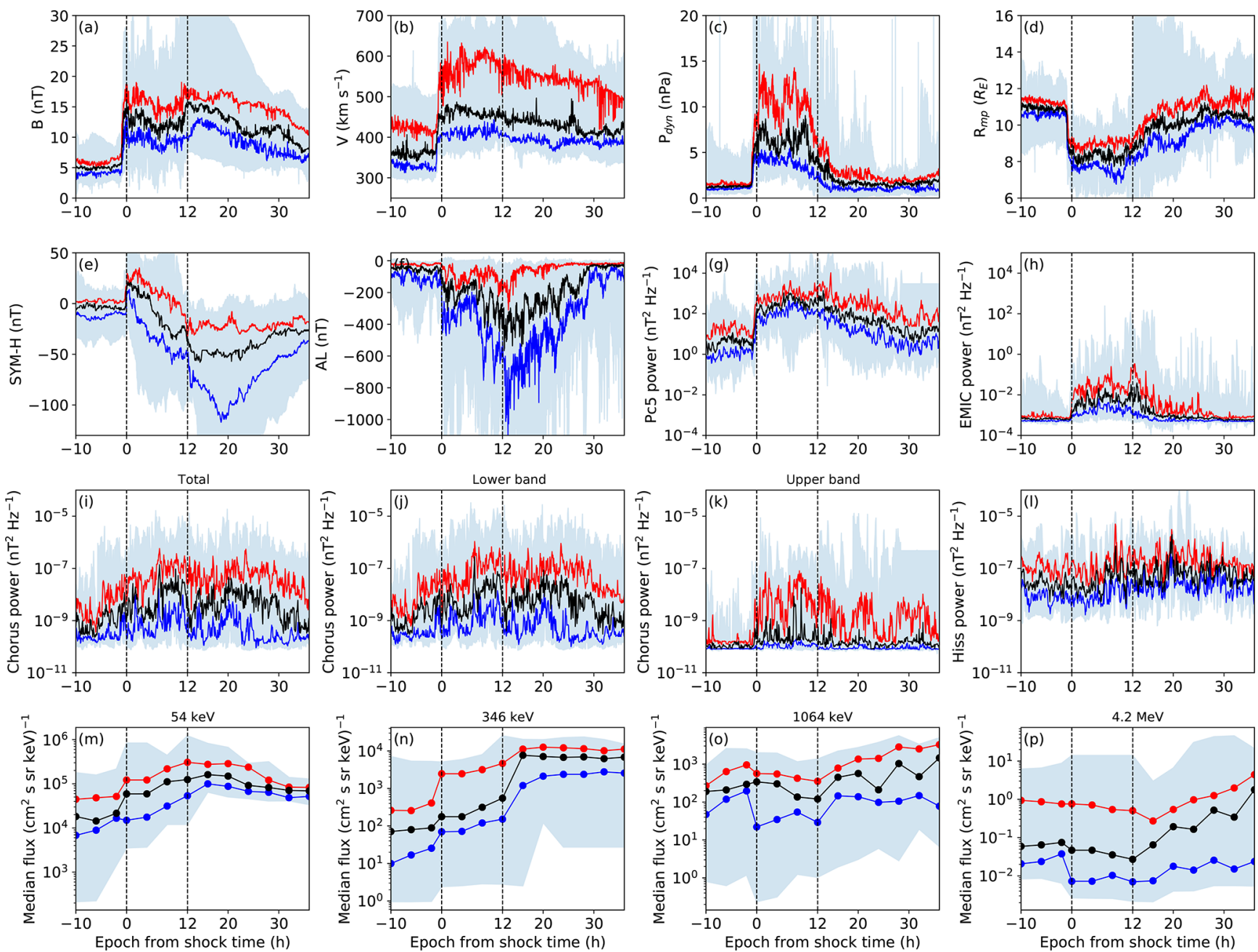

Figure 7. Same as Fig. 4 but for geoeffective sheath events where the SYM- $H$ index has a minimum of -30 nT or smaller during the sheath or $2 \mathrm{~h}$ after it. The total number of geoeffective events is 17 .

ment with a previous case study by Kilpua et al. (2019b). As discussed in Kilpua et al. (2019b), the ULF enhancement is likely due to higher and variable dynamic pressure and more turbulent variations of the magnetic field in the sheaths than in the ejecta. In this study we found enhanced ULF wave activity during sheaths, as observed inside the magnetosphere. We note that Kilpua et al. (2013) and Hietala et al. (2014) generally observed a clearly higher level of ULF-Pc5 wave power during sheaths than during the ejecta and preceding solar wind outside the magnetosphere. High ULF-Pc5 wave power in sheaths can enhance the growth rate of chorus waves (e.g. Coroniti and Kennel, 1970). However, chorus and plasmaspheric hiss wave power, in turn, had more similar levels in the sheath and ejecta. Chorus waves are excited by substorm injected electrons. Despite the clearly stronger SYM- $H$ response during the ICME ejecta, substorm activity evidenced by the AL index was comparable during the sheath and ejecta, except during about a quarter of the cases. Conse- quently, the chorus activity did not change significantly during the events.

In previous studies, the sheath response was statistically investigated at the geostationary orbit (e.g. Hietala et al., 2014; Kilpua et al., 2015) with radially resolved Van Allen Probes data spanning time periods over several days (e.g. Turner et al., 2015, 2019). In this paper we examined the immediate, and spanning a few hours, timescale sheath response over the wide $L$ shell and energy ranges more precisely. We found that sheaths deplete relativistic $\mathrm{MeV}$ electrons at higher $L$ shells (down to about $L \sim 4.5$ ). The results showed that enhancements at ultrarelativistic energies are rare, which is in agreement with a previous study by Zhao et al. (2019) that found few enhancement events of ultrarelativistic electrons during weak geomagnetic activity $\left(\right.$ Dst $\left._{\min }>-50 \mathrm{nT}\right)$ during the Van Allen Probes era. We further showed that the highest energy electrons $(>\sim 4 \mathrm{MeV})$ throughout the outer belt and $1-4 \mathrm{MeV}$ electrons in the inner part of the outer belt are mostly unchanged during the sheath 

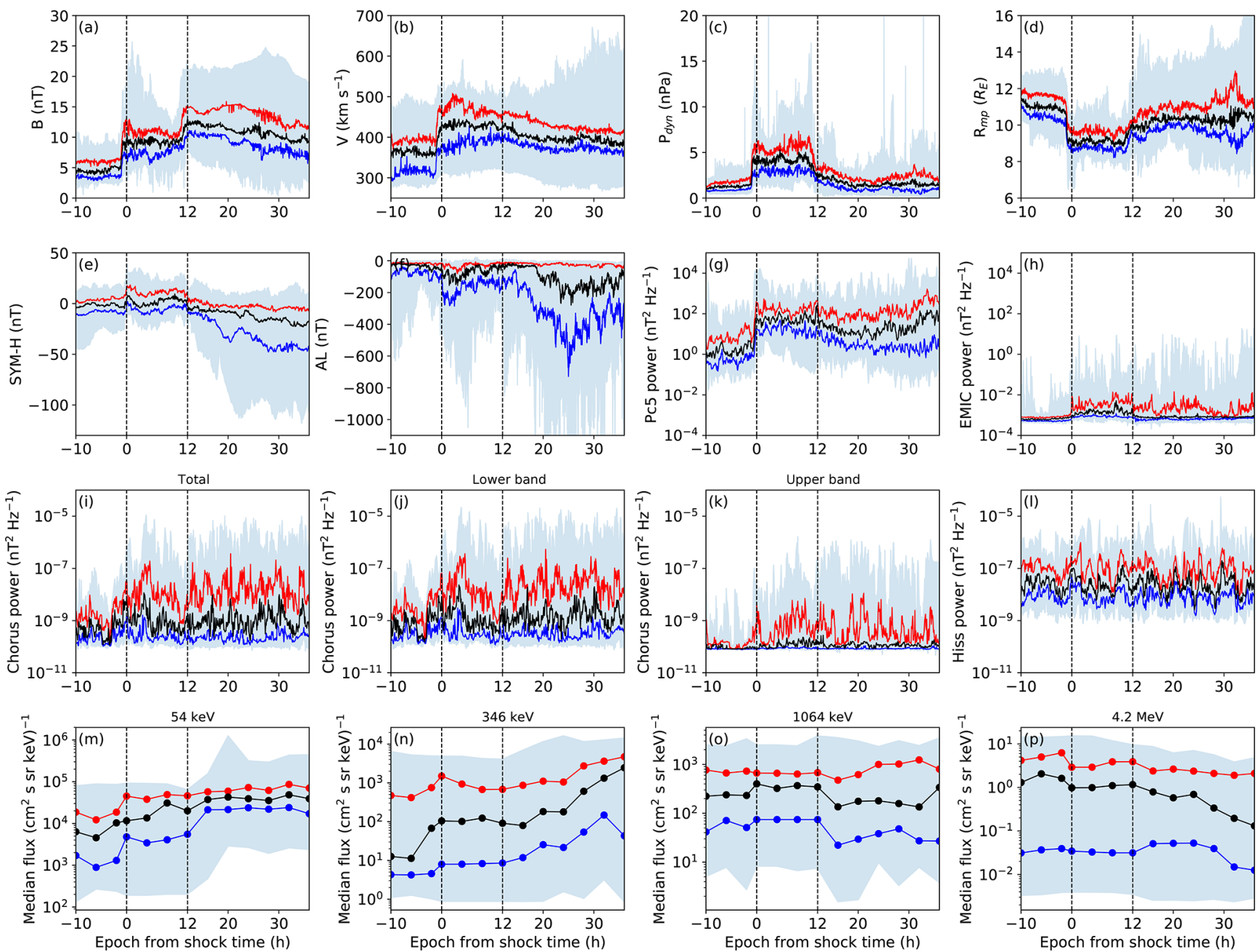

Figure 8. Same as Fig. 4 but for non-geoeffective sheath events where the SYM- $H$ index remains above $-30 \mathrm{nT}$ during the sheath and $2 \mathrm{~h}$ after it. The total number of non-geoeffective events is 20 .

passage. The source electrons (tens of keV) were in turn enhanced throughout most of the outer belt during the sheaths, despite their quite mild geoeffectiveness. In about half of the cases, seed electrons (hundreds of $\mathrm{keV}$ ) were enhanced in the heart of the outer belt, while more energetic seed electrons $(>500 \mathrm{keV})$ depleted in about half of the cases at high $L$ shells. Additionally, our example event showed that even weakly geoeffective sheaths can result in a clear outer radiation belt response up to ultrarelativistic energies in some cases. Since the sheaths cause enhancements of source and seed electrons, but mostly the depletion of most energetic seed electrons $(>500 \mathrm{keV})$ and the core population, statistically they cannot produce the so-called killer electrons $(>1-$ $2 \mathrm{MeV}$ ) in the studied timescales.

The results described previously agree on a general level with the results of ICME sheath impacts presented by Turner et al. (2019), who only considered events that caused a geomagnetic storm with a SYM- $H$ minimum below $-50 \mathrm{nT}$. Therefore, we compare their results to our results for geo- effective events only (SYM- $H$ minimum $\leq-30 \mathrm{nT}$ ). Turner et al. (2019) found that seed electrons are enhanced more often than the source population and that most enhancements occur at $L<4$, while our study revealed somewhat opposite results. The source and seed populations in our case are equally likely to be enhanced and most enhancements take place at $L>3.5$. On the other hand, Turner et al. (2019) found that depletion of $\mathrm{MeV}$ electrons was as likely throughout the outer belt, whereas we show that immediate depletion is more restricted to higher $L$ shells. The different results in our study and the one by Turner et al. (2019) are most likely attributed to the difference in the time intervals considered in these studies. We investigated the immediate sheath response in the $6 \mathrm{~h}$ before and after the sheath, while Turner et al. (2019) considered $72 \mathrm{~h}$ periods that were $12 \mathrm{~h}$ before and after the SYM- $H$ minimum. Additionally, Turner et al. (2019) only included moderate or stronger storms and used maximum flux values to calculate the response, whereas we used the median fluxes. 

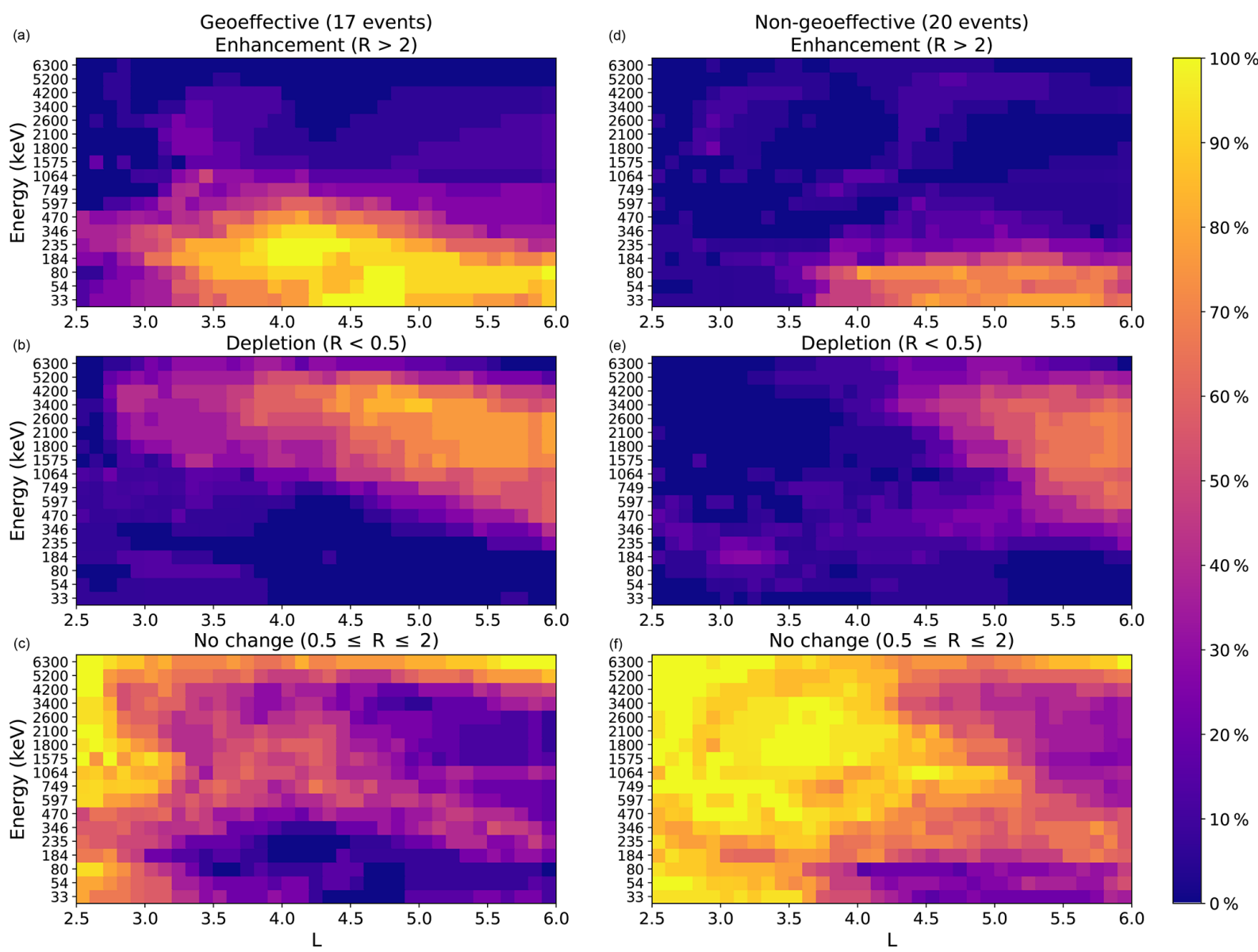

Figure 9. Percentages of sheath events causing enhancement, depletion, or no change similar to Fig. 6, with events divided based on their geoeffectiveness. Panels (a-c) show geoeffective events with a SYM- $H$ minimum of -30 nT or below. Panels (d-f) show non-geoeffective events, where SYM- $H>-30 \mathrm{nT}$.

The immediate response to the sheath has a clear energy and $L$ shell dependence. High-energy electrons cannot typically access low $L$ shells $<\sim 4$, except during strong magnetic storms and very strong solar wind drivers (e.g. Baker et al., 2014a; Reeves et al., 2016); however, ultrarelativistic electrons can also reach lower $L$ shells during weak storms via inward radial diffusion (e.g. Zhao et al., 2018; Katsavrias et al., 2019b). At low $L$ shells $(L<3.5)$ the high percentage of no-change events at lower energies $(<300 \mathrm{keV})$ is a result of the unaffected inner radiation belt population. At larger energies, no-change events at $L<3.5$ are likely due to the typically weakly populated slot region. At high $L$ shells $(L>5)$ the seed electron fluxes do not change much as the substorm injections effectively replenish the population (e.g. Turner et al., 2019). One distinct feature we found was the clear energy and $L$-shell dependence in the losses (Fig. 6b). Depletion becomes more likely when energy and $L$ shell increase but also extends to lower energies with increasing radial distance. Such dependence was not found in previous sheath response studies (e.g. Turner et al., 2019). We suggest that this energy and $L$-shell-dependent depletion can be ex- plained by the energy-dependent wave-particle interactions contributing significantly to electron losses in the heart of the outer belt, while all energies are depleted equally at larger radial distances via magnetopause shadowing that is possibly enhanced by the outward radial diffusion by ULF-Pc5 waves.

We also found the following clear differences in the wave activity and energetic electron response between geoeffective (SYM- $H$ minimum $\leq-30 \mathrm{nT}$ ) and non-geoeffective sheaths: wave activity is higher during geoeffective events, and the enhancement of the source and seed populations and the depletion of the core population are more common. In addition, a significant response also takes place at lower $L$ shells for all energies during geoeffective events (similiar to the results presented in Turner et al., 2019), while nongeoeffective events usually only cause significant changes at $L>4$. This can be attributed to geoeffective sheaths having a tendency for larger dynamic pressure, stronger ring current (SYM- $H$ ), and substorm activity (AL). Consequently, they show strong seed energy enhancement due to substorms, while $\mathrm{MeV}$ fluxes are depleted more often due to stronger 
magnetopause shadowing and possible EMIC wave scattering.

The results in this paper agree qualitatively with the general conclusions of phase-space density studies. However, we note that these studies are not quantitatively comparable with ours since we examined electron fluxes and also considered non-geoeffective events. During an intense geomagnetic storm Reeves et al. (2013) showed, by using phase space density analysis, that local acceleration, i.e. energisation via wave-particle interactions, dominated in the heart of the outer belt. Turner et al. $(2013,2014)$ showed in statistical and case studies that outer belt enhancements during geomagnetic storms are associated with local acceleration via chorus waves. Prompt depletion is consistent with magnetopause shadowing and enhanced outward radial transport, and the pitch-angle scattering by EMIC waves leads to precipitation loss (e.g. Turner et al., 2013, 2014).

In this paper, we detailed the immediate energy and $L$ shell-dependent response of the outer radiation belt to ICMEdriven sheath regions. Our comprehensive statistical analysis showed the following:

1. The inner magnetospheric wave activity is enhanced during sheaths, including those sheaths that do not cause a notable geomagnetic disturbance. Similarly, non-geoeffective sheaths can also cause a significant response in the outer belt electron fluxes. This highlights the importance of also considering events with a weak geomagnetic impact in the studies of outer radiation belt electron fluxes.

2. Electron flux enhancements occur predominantly in the heart of the outer belt at source and seed energies, while the dominant response of the core and ultrarelativistic population is depletion at high $L$ shells. Also, the higher-energy seed population is depleted at the highest sampled radial distances. These distinct results were specifically revealed, for the first time, by investigating the immediate, short timescale electron flux response.

Future work will make use of the phase space density analysis method (e.g. Green and Kivelson, 2001, 2004; Chen et al., 2005, 2007; Turner et al., 2012; Shprits et al., 2017), which excludes the effects of adiabatic processes, to study sheath response in more detail. With this method, the dominant acceleration, transport, and loss processes in the outer radiation belt during sheath regions can be better identified. With the decommissioning of the Van Allen Probes, future missions surveying the radiation belt environment through various radial distances with high energy resolution are needed for the continuous study of the near-Earth space and its response to solar wind driving. In addition to largescale missions, such as the Van Allen Probes, radiation belt missions can be realised - even with cost-effective nanosatellites (e.g. Palmroth et al., 2019).
Data availability. All RBSP-ECT data are publicly available at http://www.rbsp-ect.lanl.gov/ (RBSP-ECT Data Center, 2020), and all RBSP-EMFISIS data are publicly available at https:// emfisis.physics.uiowa.edu (RBSP-EMFISIS, 2020). GOES data were obtained from https://www.ngdc.noaa.gov/stp/satellite/goes/ index.html (NOAA, 2020), and Wind and OMNI data were obtained from CDAWeb at https://cdaweb.gsfc.nasa.gov/index.html/ (NASA, 2020).

Author contributions. MMHK carried out the data analysis, prepared the plots, and interpreted the results under the supervision of EKJK and AO. AJ helped with the interpretation of the results. MMHK prepared the paper with contributions from all the authors.

Competing interests. The authors declare that they have no conflict of interest.

Acknowledgements. The results presented here have been achieved under the framework of the Finnish Centre of Excellence in Research of Sustainable Space (FORESAIL) at the Academy of Finland, which we gratefully acknowledge. We acknowledge Harlan Spence and Geoff Reeves for the ECT data, Bern Blake for the MagEIS data, and Dan Baker for the REPT data. We are also grateful to the Van Allen Probes, GOES, Wind, and OMNI teams for making their data publicly available. The processing of the ECT, MagEIS, and REPT data was supported by the Energetic Particle, Composition, and Thermal Plasma (RBSP-ECT) investigation funded by NASA's prime contract (grant no. NAS5-01072).

Financial support. This research has been supported by the Finnish Centre of Excellence in Research of Sustainable Space (FORESAIL) at the Academy of Finland (grant no. 312390). The work of Lucile Turc was supported by the Academy of Finland (grant no. 322544).

Open access funding provided by Helsinki University Library.

Review statement. This paper was edited by Wen Li and reviewed by two anonymous referees.

\section{References}

Abel, B. and Thorne, R. M.: Electron scattering loss in Earth's inner magnetosphere 1. Dominant physical processes, J. Geophys. Res., 103, 2385-2396, https://doi.org/10.1029/97JA02919, 1998.

Albert, J. M., Ginet, G. P., and Gussenhoven, M. S.: CRRES observations of radiation belt protons 1. Data overview and steady state radial diffusion, J. Geophys. Res., 103, 9261-9274, https://doi.org/10.1029/97JA02869, 1998.

Alves, L. R., Da Silva, L. A., Souza, V. M., Sibeck, D. G., Jauer, P. R., Vieira, L. E. A., Walsh, B. M., Silveira, M. V. D., Marchezi, J. P., Rockenbach, M., Lago, A. D., Mendes, O., Tsurutani, B. T., 
Koga, D., Kanekal, S. G., Baker, D. N., Wygant, J. R., and Kletzing, C. A.: Outer radiation belt dropout dynamics following the arrival of two interplanetary coronal mass ejections, Geophys. Res. Lett., 43, 978-987, https://doi.org/10.1002/2015GL067066, 2016.

Anderson, B. R., Millan, R. M., Reeves, G. D., and Friedel, R. H. W.: Acceleration and loss of relativistic electrons during small geomagnetic storms, Geophys. Res. Lett., 42, 1011310119, https://doi.org/10.1002/2015GL066376, 2015.

Baker, D. N., Kanekal, S. G., Li, X., Monk, S. P., Goldstein, J., and Burch, J. L.: An extreme distortion of the Van Allen belt arising from the 'Hallowe'en' solar storm in 2003, Nature, 432, 878881, https://doi.org/10.1038/nature03116, 2004.

Baker, D. N., Kanekal, S. G., Hoxie, V. C., Batiste, S., Bolton, M., Li, X., Elkington, S. R., Monk, S., Reukauf, R., Steg, S., Westfall, J., Belting, C., Bolton, B., Braun, D., Cervelli, B., Hubbell, K., Kien, M., Knappmiller, S., Wade, S., Lamprecht, B., Stevens, K., Wallace, J., Yehle, A., Spence, H. E., and Friedel, R.: The Relativistic Electron-Proton Telescope (REPT) Instrument on Board the Radiation Belt Storm Probes (RBSP) Spacecraft: Characterization of Earth's Radiation Belt HighEnergy Particle Populations, Space Sci. Rev., 179, 337-381, https://doi.org/10.1007/s11214-012-9950-9, 2013.

Baker, D. N., Jaynes, A. N., Hoxie, V. C., Thorne, R. M., Foster, J. C., Li, X., Fennell, J. F., Wygant, J. R., Kanekal, S. G., Erickson, P. J., Kurth, W., Li, W., Ma, Q., Schiller, Q., Blum, L., Malaspina, D. M., Gerrard, A., and Lanzerotti, L. J.: An impenetrable barrier to ultrarelativistic electrons in the Van Allen radiation belts, Nature, 515, 531-534, https://doi.org/10.1038/nature13956, 2014a.

Baker, D. N., Jaynes, A. N., Li, X., Henderson, M. G., Kanekal, S. G., Reeves, G. D., Spence, H. E., Claudepierre, S. G., Fennell, J. F., Hudson, M. K., Thorne, R. M., Foster, J. C., Erickson, P. J., Malaspina, D. M., Wygant, J. R., Boyd, A., Kletzing, C. A., Drozdov, A., and Shprits, Y. Y.: Gradual diffusion and punctuated phase space density enhancements of highly relativistic electrons: Van Allen Probes observations, Geophys. Res. Lett., 41, 1351-1358, https://doi.org/10.1002/2013GL058942, 2014b.

Bingham, S. T., Mouikis, C. G., Kistler, L. M., Boyd, A. J., Paulson, K., Farrugia, C. J., Huang, C. L., Spence, H. E., Claudepierre, S. G., and Kletzing, C.: The Outer Radiation Belt Response to the Storm Time Development of Seed Electrons and Chorus Wave Activity During CME and CIR Driven Storms, J. Geophys. Res.-Space, 123, 10139-10157, https://doi.org/10.1029/2018JA025963, 2018.

Bingham, S. T., Mouikis, C. G., Kistler, L. M., Paulson, K. W., Farrugia, C. J., Huang, C. L., Spence, H. E., Reeves, G. D., and Kletzing, C.: The Storm Time Development of Source Electrons and Chorus Wave Activity During CME- and CIRDriven Storms, J. Geophys. Res.-Space, 124, 6438-6452, https://doi.org/10.1029/2019JA026689, 2019.

Blake, J. B., Carranza, P. A., Claudepierre, S. G., Clemmons, J. H., Crain, W. R., Dotan, Y., Fennell, J. F., Fuentes, F. H., Galvan, R. M., George, J. S., Henderson, M. G., Lalic, M., Lin, A. Y., Looper, M. D., Mabry, D. J., Mazur, J. E., McCarthy, B., Nguyen, C. Q., O’Brien, T. P., Perez, M. A., Redding, M. T., Roeder, J. L., Salvaggio, D. J., Sorensen, G. A., Spence, H. E., Yi, S., and Zakrzewski, M. P.: The Magnetic Electron Ion Spectrometer (MagEIS) Instruments Aboard the Radiation Belt
Storm Probes (RBSP) Spacecraft, Space Sci. Rev., 179, 383-421, https://doi.org/10.1007/s11214-013-9991-8, 2013.

Blum, L. W., Artemyev, A., Agapitov, O., Mourenas, D., Boardsen, S., and Schiller, Q.: EMIC Wave-Driven Bounce Resonance Scattering of Energetic Electrons in the Inner Magnetosphere, J. Geophys. Res.-Space, 124, 2484-2496, https://doi.org/10.1029/2018JA026427, 2019.

Bortnik, J. and Thorne, R. M.: The dual role of ELF/VLF chorus waves in the acceleration and precipitation of radiation belt electrons, J. Atmos. Sol.-Terr. Phy., 69, 378-386, https://doi.org/10.1016/j.jastp.2006.05.030, 2007.

Boyd, A. J., Reeves, G. D., Spence, H. E., Funsten, H. O., Larsen, B. A., Skoug, R. M., Blake, J. B., Fennell, J. F., Claudepierre, S. G., Baker, D. N., Kanekal, S. G., and Jaynes, A. N.: RBSP-ECT Combined Spin-Averaged Electron Flux Data Product, J. Geophys. Res.-Space, 124, 9124-9136, https://doi.org/10.1029/2019JA026733, 2019.

Brito, T., Woodger, L., Hudson, M., and Millan, R.: Energetic radiation belt electron precipitation showing ULF modulation, Geophys. Res. Lett., 39, L22104, https://doi.org/10.1029/2012GL053790, 2012.

Burtis, W. J. and Helliwell, R. A.: Banded chorus - A new type of VLF radiation observed in the magnetosphere by OGO 1 and OGO 3, J. Geophys. Res., 74, 3002, https://doi.org/10.1029/JA074i011p03002, 1969.

NASA: CDAWeb, available at: https://cdaweb.gsfc.nasa.gov/index. html/, last access: 1 June 2020.

Chen, Y., Friedel, R. H. W., Reeves, G. D., Onsager, T. G., and Thomsen, M. F.: Multisatellite determination of the relativistic electron phase space density at geosynchronous orbit: Methodology and results during geomagnetically quiet times, J. Geophys. Res.-Space, 110, A10210, https://doi.org/10.1029/2004JA010895, 2005.

Chen, Y., Reeves, G. D., and Friedel, R. H. W.: The energization of relativistic electrons in the outer Van Allen radiation belt, Nat. Phys., 3, 614-617, https://doi.org/10.1038/nphys655, 2007.

Claudepierre, S. G., Elkington, S. R., and Wiltberger, M.: Solar wind driving of magnetospheric ULF waves: Pulsations driven by velocity shear at the magnetopause, J. Geophys. Res.-Space, 113, A05218, https://doi.org/10.1029/2007JA012890, 2008.

Claudepierre, S. G., Hudson, M. K., Lotko, W., Lyon, J. G., and Denton, R. E.: Solar wind driving of magnetospheric ULF waves: Field line resonances driven by dynamic pressure fluctuations, J. Geophys. Res.-Space, 115, A11202, https://doi.org/10.1029/2010JA015399, 2010.

Claudepierre, S. G., O’Brien, T. P., Blake, J. B., Fennell, J. F., Roeder, J. L., Clemmons, J. H., Looper, M. D., Mazur, J. E., Mulligan, T. M., Spence, H. E., Reeves, G. D., Friedel, R. H. W., Henderson, M. G., and Larsen, B. A.: A background correction algorithm for Van Allen Probes MagEIS electron flux measurements, J. Geophys. Res.-Space, 120, 5703-5727, https://doi.org/10.1002/2015JA021171, 2015.

Coroniti, F. V. and Kennel, C. F.: Electron precipitation pulsations, J. Geophys. Res., 75, 1279-1289, https://doi.org/10.1029/JA075i007p01279, 1970.

Daglis, I. A., Katsavrias, C., and Georgiou, M.: From solar sneezing to killer electrons: outer radiation belt response to solar eruptions, Philos. T. R. Soc. A, 377, 20180097 , https://doi.org/10.1098/rsta.2018.0097, 2019. 
Engebretson, M. J., Posch, J. L., Braun, D. J., Li, W., Ma, Q., Kellerman, A. C., Huang, C. L., Kanekal, S. G., Kletzing, C. A., Wygant, J. R., Spence, H. E., Baker, D. N., Fennell, J. F., Angelopoulos, V., Singer, H. J., Lessard, M. R., Horne, R. B., Raita, T., Shiokawa, K., Rakhmatulin, R., Dmitriev, E., and Ermakova, E.: EMIC Wave Events During the Four GEM QARBM Challenge Intervals, J. Geophys. Res.-Space, 123, 6394-6423, https://doi.org/10.1029/2018JA025505, 2018.

Feynman, J. and Gabriel, S. B.: On space weather consequences and predictions, J. Geophys. Res., 105, 10543-10564, https://doi.org/10.1029/1999JA000141, 2000.

Georgiou, M., Daglis, I. A., Rae, I. J., Zesta, E., Sibeck, D. G., Mann, I. R., Balasis, G., and Tsinganos, K.: Ultralow Frequency Waves as an Intermediary for Solar Wind Energy Input Into the Radiation Belts, J. Geophys. Res.-Space, 123, 10,090-10,108, https://doi.org/10.1029/2018JA025355, 2018.

Gonzalez, W. D., Joselyn, J. A., Kamide, Y., Kroehl, H. W., Rostoker, G., Tsurutani, B. T., and Vasyliunas, V. M.: What is a geomagnetic storm?, J. Geophys. Res., 99, 5771-5792, https://doi.org/10.1029/93JA02867, 1994.

Green, J. C. and Kivelson, M. G.: A tale of two theories: How the adiabatic response and ULF waves affect relativistic electrons, J. Geophys. Res., 106, 25777-25792, https://doi.org/10.1029/2001JA000054, 2001.

Green, J. C. and Kivelson, M. G.: Relativistic electrons in the outer radiation belt: Differentiating between acceleration mechanisms, J. Geophys. Res.-Space, 109, A03213, https://doi.org/10.1029/2003JA010153, 2004.

Green, J. C., Likar, J., and Shprits, Y.: Impact of space weather on the satellite industry, Space Weather, 15, 804-818, https://doi.org/10.1002/2017SW001646, 2017.

Hands, A. D. P., Ryden, K. A., Meredith, N. P., Glauert, S. A., and Horne, R. B.: Radiation Effects on Satellites During Extreme Space Weather Events, Space Weather, 16, 1216-1226, https://doi.org/10.1029/2018SW001913, 2018.

Hartinger, M. D., Turner, D. L., Plaschke, F., Angelopoulos, V., and Singer, H.: The role of transient ion foreshock phenomena in driving Pc5 ULF wave activity, J. Geophys. Res.-Space, 118, 299-312, https://doi.org/10.1029/2012JA018349, 2013.

Hietala, H., Kilpua, E. K. J., Turner, D. L., and Angelopoulos, V.: Depleting effects of ICME-driven sheath regions on the outer electron radiation belt, Geophys. Res. Lett., 41, 2258-2265, https://doi.org/10.1002/2014GL059551, 2014.

Horne, R. B. and Pitchford, D.: Space Weather Concerns for All-Electric Propulsion Satellites, Space Weather, 13, 430-433, https://doi.org/10.1002/2015SW001198, 2015.

Iyemori, T.: Storm-time magnetospheric currents inferred from mid-latitude geomagnetic field variations, J. Geomagn. Geoelectr., 42, 1249-1265, https://doi.org/10.5636/jgg.42.1249, 1990.

Iyemori, T. and Rao, D. R. K.: Decay of the Dst field of geomagnetic disturbance after substorm onset and its implication to storm-substorm relation, Ann. Geophys., 14, 608-618, https://doi.org/10.1007/s00585-996-0608-3, 1996.

Jacobs, J. A., Kato, Y., Matsushita, S., and Troitskaya, V. A.: Classification of Geomagnetic Micropulsations, J. Geophys. Res., 69, 180-181, https://doi.org/10.1029/JZ069i001p00180, 1964.

Jaynes, A. N., Li, X., Schiller, Q. G., Blum, L. W., Tu, W., Turner, D. L., Ni, B., Bortnik, J., Baker, D. N., Kanekal, S. G., Blake,
J. B., and Wygant, J.: Evolution of relativistic outer belt electrons during an extended quiescent period, J. Geophys. Res.-Space, 119, 9558-9566, https://doi.org/10.1002/2014JA020125, 2014.

Jaynes, A. N., Baker, D. N., Singer, H. J., Rodriguez, J. V., Loto'aniu, T. M., Ali, A. F., Elkington, S. R., Li, X., Kanekal, S. G., Fennell, J. F., Li, W., Thorne, R. M., Kletzing, C. A., Spence, H. E., and Reeves, G. D.: Source and seed populations for relativistic electrons: Their roles in radiation belt changes, J. Geophys. Res.-Space, 120, 7240-7254, https://doi.org/10.1002/2015JA021234, 2015.

Jaynes, A. N., Ali, A. F., Elkington, S. R., Malaspina, D. M., Baker, D. N., Li, X., Kanekal, S. G., Henderson, M. G., Kletzing, C. A., and Wygant, J. R.: Fast Diffusion of Ultrarelativistic Electrons in the Outer Radiation Belt: 17 March 2015 Storm Event, Geophys. Res. Lett., 45, 10874-10882, https://doi.org/10.1029/2018GL079786, 2018.

Kataoka, R. and Miyoshi, Y.: Flux enhancement of radiation belt electrons during geomagnetic storms driven by coronal mass ejections and corotating interaction regions, Space Weather, 4, 09004, https://doi.org/10.1029/2005SW000211, 2006.

Katsavrias, C., Daglis, I. A., Turner, D. L., Sand berg, I., Papadimitriou, C., Georgiou, M., and Balasis, G.: Nonstorm loss of relativistic electrons in the outer radiation belt, Geophys. Res. Lett., 42, 10521-10530, https://doi.org/10.1002/2015GL066773, 2015.

Katsavrias, C., Daglis, I. A., and Li, W.: On the Statistics of Acceleration and Loss of Relativistic Electrons in the Outer Radiation Belt: A Superposed Epoch Analysis, J. Geophys. Res.Space, 124, 2755-2768, https://doi.org/10.1029/2019JA026569, 2019a.

Katsavrias, C., Sandberg, I., Li, W., Podladchikova, O., Daglis, I. A., Papadimitriou, C., Tsironis, C., and Aminalragia-Giamini, S.: Highly Relativistic Electron Flux Enhancement During the Weak Geomagnetic Storm of April-May 2017, J. Geophys. Res.Space, 124, 4402-4413, https://doi.org/10.1029/2019JA026743, 2019b.

Kepko, L. and Spence, H. E.: Observations of discrete, global magnetospheric oscillations directly driven by solar wind density variations, J. Geophys. Res.-Space, 108, 1257 https://doi.org/10.1029/2002JA009676, 2003.

Kilpua, E., Koskinen, H. E. J., and Pulkkinen, T. I.: Coronal mass ejections and their sheath regions in interplanetary space, Living Rev. Sol. Phys., 14, 5, https://doi.org/10.1007/s41116-017-00096, 2017.

Kilpua, E. K. J., Hietala, H., Koskinen, H. E. J., Fontaine, D., and Turc, L.: Magnetic field and dynamic pressure ULF fluctuations in coronal-mass-ejection-driven sheath regions, Ann. Geophys., 31, 1559-1567, https://doi.org/10.5194/angeo-31-15592013, 2013.

Kilpua, E. K. J., Hietala, H., Turner, D. L., Koskinen, H. E. J., Pulkkinen, T. I., Rodriguez, J. V., Reeves, G. D., Claudepierre, S. G., and Spence, H. E.: Unraveling the drivers of the storm time radiation belt response, Geophys. Res. Lett., 42, 30763084, https://doi.org/10.1002/2015GL063542, 2015.

Kilpua, E. K. J., Fontaine, D., Moissard, C., Ala-Lahti, M., Palmerio, E., Yordanova, E., Good, S. W., Kalliokoski, M. M. H., Lumme, E., Osmane, A., Palmroth, M., and Turc, L.: Solar wind properties and geospace impact of coronal mass ejection-driven 
sheath regions: Variation and driver dependence, Space Weather, 17, 1257-1280, https://doi.org/10.1029/2019SW002217, 2019a.

Kilpua, E. K. J., Turner, D. L., Jaynes, A. N., Hietala, H., Koskinen, H. E. J., Osmane, A., Palmroth, M., Pulkkinen, T. I., Vainio, R., Baker, D., and Claudepierre, S. G.: Outer Van Allen Radiation Belt Response to Interacting Interplanetary Coronal Mass Ejections, J. Geophys. Res.-Space, 124, 1927-1947, https://doi.org/10.1029/2018JA026238, 2019b.

Kletzing, C. A., Kurth, W. S., Acuna, M., MacDowall, R. J., Torbert, R. B., Averkamp, T., Bodet, D., Bounds, S. R., Chutter, M., Connerney, J., Crawford, D., Dolan, J. S., Dvorsky, R., Hospodarsky, G. B., Howard, J., Jordanova, V., Johnson, R. A., Kirchner, D. L., Mokrzycki, B., Needell, G., Odom, J., Mark, D., Pfaff, R., Phillips, J. R., Piker, C. W., Remington, S. L., Rowland, D., Santolik, O., Schnurr, R., Sheppard, D., Smith, C. W., Thorne, R. M., and Tyler, J.: The Electric and Magnetic Field Instrument Suite and Integrated Science (EMFISIS) on RBSP, Space Sci. Rev., 179, 127-181, https://doi.org/10.1007/s11214-013-9993-6, 2013.

Koons, H. C. and Roeder, J. L.: A survey of equatorial magnetospheric wave activity between 5 and $8 R_{\mathrm{E}}$, Planet Space Sci., 38, 1335-1341, https://doi.org/10.1016/0032-0633(90)90136-E, 1990.

Kurth, W. S., De Pascuale, S., Faden, J. B., Kletzing, C. A., Hospodarsky, G. B., Thaller, S., and Wygant, J. R.: Electron densities inferred from plasma wave spectra obtained by the Waves instrument on Van Allen Probes, J. Geophys. Res.-Space, 120, 904 914, https://doi.org/10.1002/2014JA020857, 2015.

Lepping, R. P., Acũna, M. H., Burlaga, L. F., Farrell, W. M., Slavin, J. A., Schatten, K. H., Mariani, F., Ness, N. F., Neubauer, F. M., Whang, Y. C., Byrnes, J. B., Kennon, R. S., Panetta, P. V., Scheifele, J., and Worley, E. M.: The Wind Magnetic Field Investigation, Space Sci. Rev., 71, 207-229, https://doi.org/10.1007/BF00751330, 1995.

Lugaz, N., Farrugia, C. J., Winslow, R. M., Al-Haddad, N., Kilpua, E. K. J., and Riley, P.: Factors affecting the geoeffectiveness of shocks and sheaths at $1 \mathrm{AU}, \mathrm{J}$. Geophys. Res.-Space, 121, 1086110879, https://doi.org/10.1002/2016JA023100, 2016.

Masías-Meza, J. J., Dasso, S., Démoulin, P., Rodriguez, L., and Janvier, M.: Superposed epoch study of ICME substructures near Earth and their effects on Galactic cosmic rays, Astron. Astrophys., 592, A118, https://doi.org/10.1051/00046361/201628571, 2016.

Mauk, B. H., Fox, N. J., Kanekal, S. G., Kessel, R. L., Sibeck, D. G., and Ukhorskiy, A.: Science Objectives and Rationale for the Radiation Belt Storm Probes Mission, Space Sci. Rev., 179, 3-27, https://doi.org/10.1007/s11214-012-9908-y, 2013.

McIlwain, C. E.: Coordinates for Mapping the Distribution of Magnetically Trapped Particles, J. Geophys. Res., 66, 3681-3691, https://doi.org/10.1029/JZ066i011p03681, 1961.

Moya, P. S., Pinto, V. A., Sibeck, D. G., Kanekal, S. G., and Baker, D. N.: On the Effect of Geomagnetic Storms on Relativistic Electrons in the Outer Radiation Belt: Van Allen Probes Observations, J. Geophys. Res.-Space, 122, 1110011108, https://doi.org/10.1002/2017JA024735, 2017.

Murphy, K. R., Watt, C. E. J., Mann, I. R., Jonathan Rae, I., Sibeck, D. G., Boyd, A. J., Forsyth, C. F., Turner, D. L., Claudepierre, S. G., Baker, D. N., Spence, H. E., Reeves, G. D., Blake, J. B., and Fennell, J.: The Global Statistical Response of the Outer Ra- diation Belt During Geomagnetic Storms, Geophys. Res. Lett., 45, 3783-3792, https://doi.org/10.1002/2017GL076674, 2018.

NOAA: GOES Data Access, available at: https://www.ngdc.noaa. gov/stp/satellite/goes/dataaccess.html, last access: 1 June 2020.

O'Brien, T. P. and Moldwin, M. B.: Empirical plasmapause models from magnetic indices, Geophys. Res. Lett., 30, 1152 https://doi.org/10.1029/2002GL016007, 2003.

O’Brien, T. P., McPherron, R. L., Sornette, D., Reeves, G. D., Friedel, R., and Singer, H. J.: Which magnetic storms produce relativistic electrons at geosynchronous orbit?, J. Geophys. Res., 106, 15533-15544, https://doi.org/10.1029/2001JA000052, 2001.

Ogilvie, K. W., Chornay, D. J., Fritzenreiter, R. J., Hunsaker, F., Keller, J., Lobell, J., Miller, G., Scudder, J. D., Sittler Jr., E. C., Torbert, R. B., Bodet, D., Needell, G., Lazarus, A. J., Steinberg, J. T., Tappan, J. H., Mavretic, A., and Gergin, E.: SWE, A Comprehensive Plasma Instrument for the Wind Spacecraft, Space Sci. Rev., 71, 55-77, https://doi.org/10.1007/BF00751326, 1995.

Palmerio, E., Kilpua, E. K. J., and Savani, N. P.: Planar magnetic structures in coronal mass ejection-driven sheath regions, Ann. Geophys., 34, 313-322, https://doi.org/10.5194/angeo-34-3132016, 2016.

Palmroth, M., Praks, J., Vainio, R., Janhunen, P., Kilpua, E. K. J., Ganushkina, N. Y., Afanasiev, A., Ala-Lahti, M., Alho, A., Asikainen, T., Asvestari, E., Battarbee, M., Binios, A., Bosser, A., Brito, T., Envall, J., Ganse, U., George, H., Gieseler, J., Good, S., Grand in, M., Haslam, S., Hedman, H. P., Hietala, H., Jovanovic, N., Kakakhel, S., Kalliokoski, M., Kettunen, V. V., Koskela, T., Lumme, E., Meskanen, M., Morosan, D., Rizwan Mughal, M., Niemelä, P., Nyman, S., Oleynik, P., Osmane, A., Palmerio, E., Pfau-Kempf, Y., Peltonen, J., Plosila, J., Polkko, J., Poluianov, S., Pomoell, J., Price, D., Punkkinen, A., Punkkinen, R., Riwanto, B., Salomaa, L., Slavinskis, A., Säntti, T., Tammi, J., Tenhunen, H., Toivanen, P., Tuominen, J., Turc, L., Valtonen, E., Virtanen, P., and Westerlund, T.: FORESAIL-1 cubesat mission to measure radiation belt losses and demonstrate deorbiting, J. Geophys. Res.-Space, 124, 57835799, https://doi.org/10.1029/2018JA026354, 2019.

RBSP-ECT Data Center: RBSP-ECT Science Data Products, LANL, available at: https://www.rbsp-ect.lanl.gov/science/ DataDirectories.php, last access: 1 June 2020.

RBSP-EMFISIS: Data Index, University of Iowa, available at: https://emfisis.physics.uiowa.edu/data/index, last access: 1 June 2020.

Reeves, G. D., McAdams, K. L., Friedel, R. H. W., and O'Brien, T. P.: Acceleration and loss of relativistic electrons during geomagnetic storms, Geophys. Res. Lett., 30, 1529, https://doi.org/10.1029/2002GL016513, 2003.

Reeves, G. D., Spence, H. E., Henderson, M. G., Morley, S. K., Friedel, R. H. W., Funsten, H. O., Baker, D. N., Kanekal, S. G., Blake, J. B., Fennell, J. F., Claudepierre, S. G., Thorne, R. M., Turner, D. L., Kletzing, C. A., Kurth, W. S., Larsen, B. A., and Niehof, J. T.: Electron Acceleration in the Heart of the Van Allen Radiation Belts, Science, 341, 991-994, https://doi.org/10.1126/science.1237743, 2013.

Reeves, G. D., Friedel, R. H. W., Larsen, B. A., Skoug, R. M., Funsten, H. O., Claudepierre, S. G., Fennell, J. F., Turner, D. L., Denton, M. H., Spence, H. E., Blake, J. B., and Baker, D. N.: Energy-dependent dynamics of $\mathrm{keV}$ to $\mathrm{MeV}$ electrons in the in- 
ner zone, outer zone, and slot regions, J. Geophys. Res.-Space, 121, 397-412, https://doi.org/10.1002/2015JA021569, 2016.

Richardson, I. G. and Cane, H. V.: Near-Earth Interplanetary Coronal Mass Ejections During Solar Cycle 23 (1996-2009): Catalog and Summary of Properties, Sol. Phys., 264, 189-237, https://doi.org/10.1007/s11207-010-9568-6, 2010.

Schiller, Q., Li, X., Blum, L., Tu, W., Turner, D. L., and Blake, J. B.: A nonstorm time enhancement of relativistic electrons in the outer radiation belt, Geophys. Res. Lett., 41, 7-12, https://doi.org/10.1002/2013GL058485, 2014.

Selesnick, R. S., Baker, D. N., Jaynes, A. N., Li, X., Kanekal, S. G., Hudson, M. K., and Kress, B. T.: Inward diffusion and loss of radiation belt protons, J. Geophys. Res.-Space, 121, 1969-1978, https://doi.org/10.1002/2015JA022154, 2016.

Shprits, Y. Y., Thorne, R. M., Friedel, R., Reeves, G. D., Fennell, J., Baker, D. N., and Kanekal, S. G.: Outward radial diffusion driven by losses at magnetopause, J. Geophys. Res.-Space, 111, A11214, https://doi.org/10.1029/2006JA011657, 2006.

Shprits, Y. Y., Kellerman, A., Aseev, N., Drozdov, A. Y., and Michaelis, I.: Multi-MeV electron loss in the heart of the radiation belts, Geophys. Res. Lett., 44, 1204-1209, https://doi.org/10.1002/2016GL072258, 2017.

Shue, J. H., Song, P., Russell, C. T., Steinberg, J. T., Chao, J. K., Zastenker, G., Vaisberg, O. L., Kokubun, S., Singer, H. J., and Detman, T. R.: Magnetopause location under extreme solar wind conditions, J. Geophys. Res., 103, 17691-17700, https://doi.org/10.1029/98JA01103, 1998.

Singer, H., Matheson, L., Grubb, R., Newman, A., and Bouwer, D.: Monitoring space weather with the GOES magnetometers, in: GOES-8 and Beyond, edited by: Washwell, E. R., Vol. 2812 of Society of Photo-Optical Instrumentation Engineers (SPIE) Conference Series, 299-308, https://doi.org/10.1117/12.254077, International Society for Optics and Photonics, Denver, CO, USA, 1996.

Spence, H. E., Reeves, G. D., Baker, D. N., Blake, J. B., Bolton, M., Bourdarie, S., Chan, A. A., Claudepierre, S. G., Clemmons, J. H., Cravens, J. P., Elkington, S. R., Fennell, J. F., Friedel, R. H. W., Funsten, H. O., Goldstein, J., Green, J. C., Guthrie, A., Henderson, M. G., Horne, R. B., Hudson, M. K., Jahn, J.-M., Jordanova, V. K., Kanekal, S. G., Klatt, B. W., Larsen, B. A., Li, X., MacDonald, E. A., Mann, I. R., Niehof, J., O'Brien, T. P., Onsager, T. G., Salvaggio, D., Skoug, R. M., Smith, S. S., Suther, L. L., Thomsen, M. F., and Thorne, R. M.: Science Goals and Overview of the Radiation Belt Storm Probes (RBSP) Energetic Particle, Composition, and Thermal Plasma (ECT) Suite on NASA's Van Allen Probes Mission, Space Sci. Rev., 179, 311336, https://doi.org/10.1007/s11214-013-0007-5, 2013.

Su, Z., Zhu, H., Xiao, F., Zong, Q. G., Zhou, X. Z., Zheng, H., Wang, Y., Wang, S., Hao, Y. X., Gao, Z., He, Z., Baker, D. N., Spence, H. E., Reeves, G. D., Blake, J. B., and Wygant, J. R.: Ultra-low-frequency wave-driven diffusion of radiation belt relativistic electrons, Nat. Commun., 6, 10096, https://doi.org/10.1038/ncomms10096, 2015.

Thorne, R. M.: Radiation belt dynamics: The importance of wave-particle interactions, Geophys. Res. Lett., 37, L22107, https://doi.org/10.1029/2010GL044990, 2010.

Tsyganenko, N. A. and Sitnov, M. I.: Modeling the dynamics of the inner magnetosphere during strong geo- magnetic storms, J. Geophys. Res.-Space, 110, A03208, https://doi.org/10.1029/2004JA010798, 2005.

Turner, D. L., Shprits, Y., Hartinger, M., and Angelopoulos, V.: Explaining sudden losses of outer radiation belt electrons during geomagnetic storms, Nat. Phys., 8, 208-212, https://doi.org/10.1038/nphys2185, 2012.

Turner, D. L., Angelopoulos, V., Li, W., Hartinger, M. D., Usanova, M., Mann, I. R., Bortnik, J., and Shprits, Y.: On the storm-time evolution of relativistic electron phase space density in Earth's outer radiation belt, J. Geophys. Res.-Space, 118, 2196-2212, https://doi.org/10.1002/jgra.50151, 2013.

Turner, D. L., Angelopoulos, V., Li, W., Bortnik, J., Ni, B., Ma, Q., Thorne, R. M., Morley, S. K., Henderson, M. G., Reeves, G. D., Usanova, M., Mann, I. R., Claudepierre, S. G., Blake, J. B., Baker, D. N., Huang, C. L., Spence, H., Kurth, W., Kletzing, C., and Rodriguez, J. V.: Competing source and loss mechanisms due to wave-particle interactions in Earth's outer radiation belt during the 30 September to 3 October 2012 geomagnetic storm, J. Geophys. Res.-Space, 119, 1960-1979, https://doi.org/10.1002/2014JA019770, 2014.

Turner, D. L., O'Brien, T. P., Fennell, J. F., Claudepierre, S. G., Blake, J. B., Kilpua, E. K. J., and Hietala, H.: The effects of geomagnetic storms on electrons in Earth's radiation belts, Geophys. Res. Lett., 42, 9176-9184, https://doi.org/10.1002/2015GL064747, 2015.

Turner, D. L., Kilpua, E. K. J., Hietala, H., Claudepierre, S. G., O’Brien, T. P., Fennell, J. F., Blake, J. B., Jaynes, A. N., Kanekal, S., Baker, D. N., Spence, H. E., Ripoll, J.-F., and Reeves, G. D.: The Response of Earth's Electron Radiation Belts to Geomagnetic Storms: Statistics From the Van Allen Probes Era Including Effects From Different Storm Drivers, J. Geophys. Res.-Space, 124, 1013-1034, https://doi.org/10.1029/2018JA026066, 2019.

Usanova, M. E., Drozdov, A., Orlova, K., Mann, I. R., Shprits, Y., Robertson, M. T., Turner, D. L., Milling, D. K., Kale, A., and Baker, D. N.: Effect of EMIC waves on relativistic and ultrarelativistic electron populations: Ground-based and Van Allen Probes observations, Geophys. Res. Lett., 41, 1375-1381, https://doi.org/10.1002/2013GL059024, 2014.

Van Allen, J. A.: The Geomagnetically Trapped Corpuscular Radiation, J. Geophys. Res., 64, 1683-1689, https://doi.org/10.1029/JZ064i011p01683, 1959.

Wang, C.-P., Thorne, R., Liu, T. Z., Hartinger, M. D., Nagai, T., Angelopoulos, V., Wygant, J. R., Breneman, A., Kletzing, C., Reeves, G. D., Claudepierre, S. G., and Spence, H. E.: A multispacecraft event study of Pc5 ultralow-frequency waves in the magnetosphere and their external drivers, J. Geophys. Res.Space, 122, 5132-5147, https://doi.org/10.1002/2016JA023610, 2017.

Yermolaev, Y. I., Lodkina, I. G., and Yermolaev, M. Y.: Dynamics of Large-Scale Solar-Wind Streams Obtained by the Double Superposed Epoch Analysis: 3. Deflection of the Velocity Vector, Sol. Phys., 293, 91, https://doi.org/10.1007/s11207-018-1310-9, 2018.

Zhang, X. J., Mourenas, D., Artemyev, A. V., Angelopoulos, V., and Sauvaud, J. A.: Precipitation of $\mathrm{MeV}$ and Sub-MeV Electrons Due to Combined Effects of EMIC and ULF Waves, J. Geophys. Res.-Space, 124, 7923-7935, https://doi.org/10.1029/2019JA026566, 2019. 
Zhao, H., Baker, D. N., Li, X., Jaynes, A. N., and Kanekal, S. G.: The Acceleration of Ultrarelativistic Electrons During a Small to Moderate Storm of 21 April 2017, Geophys. Res. Lett., 45, 5818-5825, https://doi.org/10.1029/2018GL078582, 2018.
Zhao, H., Baker, D. N., Li, X., Jaynes, A. N., and Kanekal, S. G.: The Effects of Geomagnetic Storms and Solar Wind Conditions on the Ultrarelativistic Electron Flux Enhancements, J. Geophys. Res.-Space, 124, 1948-1965, https://doi.org/10.1029/2018JA026257, 2019. 